\title{
Classification biologique des lacs jurassiens à l'aide d'une nouvelle méthode d'analyse des peuplements benthiques' I. Variété et densité de la faune
}

\author{
J. Verneaux ${ }^{2}$ \\ V. Verneaux ${ }^{2}$ \\ A. Guyard2
}

Mots clés : lacs, sédiments, macrobenthos, échantillonnage, typologie.

Les auteurs proposent une nouvelle méthode de classification biologique des lacs fondée sur une.analyse comparative de la macrofaune des sédiments.

Cette recherche inclut la définition préalable d'un protocole d'échantillonnage permettant la comparaison de systèmes lacustres de profondeur maximale très différente. Ce protocole original est caractérisé par :

- des prélèvements de sédiments meubles effectués à une profondeur en zone littorale $\left(Z_{0}: 1-2 \mathrm{~m}\right)$ et à trois profondeurs, relatives à la profondeur maximale des systèmes étudiés, en zone limnétique $\left(Z_{1}=0,25 \mathrm{Zmax}, Z_{2}=0,50 \mathrm{Zmax}\right.$, $\left.\mathrm{Z}_{3}=0,75 \mathrm{Zmax}\right)$;

- un nombre d'échantillons par profondeur prospectée (ni) proportionnel à la longueur ( $\mathrm{L}$ en $\mathrm{km})$ de chaque isobathe $\left(\mathrm{n}_{0}=1,8 \sqrt{10 \mathrm{~L}}\right.$ et $\left.\mathrm{n}_{1}, \mathrm{n}_{2}, \mathrm{n}_{3}=1,4 \sqrt{10 \mathrm{~L}}\right)$.

325 stations ont été réparties, suivant un protocole standard, dans 9 lacs du Jura et 104 taxons, dont 98 genres ont été recensés.

Une analyse factorielle des correspondances, appliquée aux stations et aux genres, montre que les stations se distribuent le long de l'axe $\mathrm{F}_{1}$, dans un ordre de variété faunistique décroissante et de profondeur croissante, exprimant l'évolution de la variété et de la densité en fonction des modifications des conditions écologiques avec la profondeur.

Une première différenciation des lacs a été obtenue en fonction de la variété totale et de la densité globale des 9 systèmes étudiés. Ces deux critères permettent de distinguer des lacs très biogènes et des lacs peu biogènes (variété totale et densité globale faibles). Cette deuxième catégorie de lacs regroupant toutefois des systèmes dont les caractéristiques écologiques différaient nettement, une seconde classification biologique a été établie par la prise en compte :

- d'un critère quantitatif : le couple variété-densité en zone littorale exprimant le potentiel biogénique des systèmes $\left(B_{o}=v_{o} \sqrt{d_{o}}\right)$;

- d'un critère qualitatif : la régression de la variété générique en fonction de la profondeur relative. Une hypothèse de linéarité de la distribution bathymétrique relative de la variété faunistique permet le calcul d'indices de distribution faunistique $\left(f_{i}=k_{i} v_{i} / v_{0}\right)$ qui traduisent l'évolution des conditions écologiques avec la profondeur.

Ces deux critères $\left(B_{o}\right.$ et $\left.f\right)$ constituent les coordonnées d'un système d'axes comportant 4 niveaux ( 2 niveaux quantitatifs et 2 niveaux qualitatifs) qui permettent de classer les lacs en fonction de leur aptitude biogénique.

Biological classification of 9 lakes of the French Jura using a new comparative analysis of the macrobenthic populations I. Variety and density of the fauna

Keywords : lakes, sediments, macrobenthos, sampling method, typology.

The authors propose a new biological classification of lakes based on a comparative analysis of sediment macrofauna.

It was first necessary to state clearly a sampling method that allows a comparison between lakes which have very different maximum depths.

This original sampling method is characterised by :

- sampling of fine sediments at one littoral depth $\left(\mathrm{Z}_{\mathrm{o}}=1,2 \mathrm{~m}\right)$ and at three depths, relative to maximum depth, on the limnetic area $\left(Z_{1}=0,25 \mathrm{Zmax}, \mathrm{Z}_{2}=0,50 \mathrm{Zmax}, \mathrm{Z}_{3}=0,75 \max \right)$.

- a number of samples, at any particular depth, proportional to the length of the particular isobath $\left(\mathrm{n}_{\mathrm{o}}=\right.$ $\left.1,8 \sqrt{10 L}, n_{1}, n_{2}, n_{3}=1,4 \sqrt{10 L}\right)$

1. Programme soutenu par le Conseil Régional de Franche-Comté et l'Agence de l'Eau Rhône-Méditerranée-Corse.

2. Laboratoire d'Hydrobiologie-Hydroécologie de l'Institut des Sciences et des Techniques de l'Environnement (I.S.T.E.) de l'Université de Franche-Comté, Place Leclerc, 25030 Besançon Cedex. 
According to this protocol, we have collected 104 taxa including 98 genera from 325 stations distributed in 9 lakes of the Jura.

A factorial correspondence analysis, applied to stations and genera, shows that stations are distributed along the first axis according to decreasing variety and increasing depth.

This axis is the expression of the changes in variety and density, depending on transformations of the physico-chemical characteristics with depth.

Then we realised a first discrimination between lakes based on the analysis of the total variety and density of the 9 studied systems.

These two criteria have facilitated a separation of the lakes into those with high variety and density (biogenic lakes) and those with low variety and density (less biogenic lakes).

Because this second group of systems was constituted by lakes with very different ecological characteristics, a second biological classification has been made considering :

- a quantitative criterion : the littoral variety-density coupling which will express the biogenic potential of the lakes $\left(B_{0}=\right.$ vo $\sqrt{\text { do })}$.

- a quantitative criterion : the decrease of the generic variety with the relative depth.

A linearity hypothesis of the relative bathymetric distribution of the faunistic variety allows the calculation of indexes of faunistic distribution ( $\mathrm{fi}=\mathrm{kivi} / \mathrm{vo}$ ) which are related to the bathymetric evolution of ecological conditions.

Thus we obtain an axis system based on these two criteria $\left(B_{o}\right.$ and $f$ ), which is composed of four levels (two quantitative levels and two qualitative levels) in which lakes are distributed according to their biogenic rates.

\section{Introduction}

Le présent essai s'inscrit dans le cadre général des recherches sur la biotypologie des systèmes lacustres.

La majorité des travaux effectués dans ce domaine, que l'on peut jalonner par les propositions de Thienemann (1913-1931), Brundin (1949-1958) et Saether (1975) les plus souvent prises comme références, se fonde sur la présence ou l'absence d'espèces, ou de groupements d'espèces considérées comme « indicatrices ».

Notre approche diffère dans la mesure où, s'appuyant sur la structure physico-chimique verticale des lacs se traduisant par une simplification des biocénoses benthiques avec la profondeur, elle est fondée sur une analyse des deux critères de base de l'analyse des peuplements : la variété et la densité.

Cette démarche s'inscrit à la suite des travaux de Lündbeck (1926-1936), de Humphries (1936) et des auteurs qui suivirent, dont les principaux résultats sur les distributions bathymétriques des macroinvertébrés figurent dans la synthèse de Brinkhurst (1974).

L'analyse bibliographique met en évidence :

- le petit nombre des travaux consacrés à la variété faunistique, la plupart des analyses comparatives prenant en compte la densité ou, plus récemment, des indices de diversité ;
- la grande inégalité des efforts d'échantillonnage exercés sur les différentes zones ou strates d'un même lac et d'un lac à un autre ;

- la faible signification que présente la comparaison des faunes relevées à égale profondeur dans des systèmes dont les profondeurs maximales sont nettement différentes.

On observe sur l'ensemble des travaux de biotypologie lacustre que, si les identifications sont souvent à l'espèce, les protocoles d'échantillonnage témoignent d'une grande diversité et parfois d'une grande imprécision. Cette disparité rend problématique la confrontation des données physicochimiques et biologiques obtenues par des opérateurs différents sur les mêmes lacs et, a fortiori, sur des systèmes différents. Si plusieurs ouvrages de synthèse traitent des techniques d'échantillonnage et de l'analyse des échantillons de la macrofaune benthique (Cummins 1962, Schwoerbel 1966, Elliott 1977, Downing \& Rigler 1984), nous n'avons pas trouvé de données relatives à un protocole d'échantillonnage adapté à l'étude comparative des lacs.

Il faut cependant mentionner qu'un essai d'adaptation du nombre des relevés à l'importance des plans d'eau a été effectué par Hakanson (1981) qui propose, pour les analyses sédimentologiques, d'effectuer $\mathrm{n}$ échantillons $=2.5+\sqrt{\text { A.F./2 avec }}$ $\mathrm{A}=$ surface du lac en $\mathrm{km}^{2}$ et $\mathrm{F}=$ développement 
littoral en $\mathrm{km}$. L'application de cette formule conduirait à réaliser 7 échantillons au lac de StPoint, le plus vaste des lacs du Jura $(A \simeq 400 \mathrm{ha})$.

A ces données initiales s'ajoute le caractère normalement contagieux de la distribution des organismes benthiques, et vérifié dans le cas des lacs étudiés $\left(1.4<\mathrm{S}^{2} / \overline{\mathrm{X}}<8.5\right.$ pour la variété générique et $3.7<\mathrm{S}^{2} / \overline{\mathrm{X}}<72.5$ pour la densité -4 lacs, 6 profondeurs -240 échantillons). Partant de ces bases, nous nous sommes proposés de rechercher les conditions expérimentales et les critères d'une différenciation biologique des lacs sans qu'il soit fait référence à la nature des organismes dont le caractère indicateur sera examiné ultérieurement.
L'expérimentation porte sur 926 échantillons prélevés à 9 profondeurs différentes dans 14 lacs de la Franche-Comté jurassienne. L'objectif poursuivi impliquait la définition préalable d'un protocole d'analyse comparative de la macrofaune benthique, celui-ci constituant un élément essentiel de la démarche effectuée.

\section{Lacs étudiés, matériel et technique}

\subsection{Les lacs}

La situation géographique et les principales caractéristiques physiques et chimiques des lacs étudiés ont été exposées dans des publications antérieures (Verneaux et al. 1987-1988-1991) ; les données utilisées dans les commentaires figurent dans le tableau 1.

Tableau 1. Quelques caractéristiques des lacs jurassiens. Ab : Abbaye, Bo : Bonlieu, Ch : Châlain, Cl : Clairvaux, Il : Ilay, Ma : Maclu, $\mathrm{Na}$ : Narlay, Re : Remoray, Sp : St. Point. $\mathrm{x}$ fond = valeur moyenne de 10 échantillons de sédiments prélevés sur l'isobathe profonde.

Table 1. Some characteristics of the lakes of the Jura. Ab : Abbaye, Bo : Bonlieu, Ch : Châlain, Cl : Clairvaux, Il : Ilay, Ma : Maclu, $\mathrm{Na}$ : Narlay, $\mathrm{Re}$ : Remoray, Sp : St-Point. $\mathrm{x}$ bottom : mean value of 10 samples of sediments, taken at the deep isobath.

\begin{tabular}{|c|c|c|c|c|c|c|c|c|c|}
\hline & $A b$ & Bo & Ch & $\mathrm{Cl}$ & II & $\mathrm{Ma}$ & $\mathrm{Na}$ & $\mathbf{P}_{\theta}$ & Sp \\
\hline alt $(m)$ & 910 & 803 & 450 & 534 & 778 & 779 & 750 & 850 & 850 \\
\hline A (ha) & 80 & 17 & 232 & 56 & 72 & 22 & 40 & 95 & 419 \\
\hline $\mathrm{Zm}(\mathrm{m})$ & 18 & 15 & 32 & 20 & 32 & 24 & 38 & 28 & 41 \\
\hline $\max$ & 260 & 250 & 360 & 360 & 250 & 255 & 260 & 355 & 335 \\
\hline \multicolumn{10}{|l|}{ C $(\mu S / \mathrm{cm})$} \\
\hline $\min$ & 150 & 165 & 175 & 215 & 145 & 150 & 180 & 210 & 220 \\
\hline \multicolumn{10}{|l|}{ MO ś́dim. } \\
\hline $\bar{x}$ fond (\%ms) & 32 & 35 & 7 & 5 & 29 & 17 & 18 & 15 & 10 \\
\hline \multicolumn{10}{|l|}{ CaCO3 sédim. } \\
\hline $\bar{x}$ fond $(\% \mathrm{~ms})$ & 40 & 34 & 80 & 83 & 62 & 70 & 75 & 74 & 78 \\
\hline
\end{tabular}

Les lacs étudiés, pour la plupart d'origine tectonoglaciaire et de profondeur moyenne, appartiennent au type bicarbonaté-calcique, à potentiel minéral élevé, mais se différencient nettement par la teneur en matière organique des sédiments.

\subsection{Technique d'échantillonnage et matériel utilisé}

\section{Substrat prospecté}

Afin d'éviter autant que possible l'introduction d'une variabilité imputable à la nature de l'habitat, pouvant être très grande en zone littorale, les analyses sont effectuées sur un seul type de substrat : les sédiments fins qui constituent les « vases » lacustres.

Les macrophytes sont évités ainsi que les secteurs particuliers comme les zones soumises à l'influence directe de vecteurs fluviatiles ou d'effluents d'eaux résiduaires, les plages aménagées ou les ports.
Dans ces conditions, la macrofaune considérée, épi- et surtout endo-benthique, constitue la synusie des sédiments fins.

Le tableau 2 met en évidence le fait, déterminant dans l'hypothèse de régression linéaire émise plus loin, que l'écart entre la grande variété de la faune littorale et celle, nettement plus réduite, de la faune profonde, s'atténue sensiblement lorsque l'on se limite à la prospection des fonds meubles. De ce fait, la comparaison des biocénoses échappe, dans une certaine mesure, à la variabilité inhérente à l'hétérogénéité des mosaïques d'habitats littoraux. L'analyse biocénotique comparative peut alors être effectuée dans des conditions habitationnelles similaires sinon identiques.

\section{Appareil}

Les prélèvements ont été réalisés à l'aide d'une benne de type Ekman, de $215 \mathrm{~cm}^{2}$ de surface, et modifiée de façon à réduire le prélèvement aux cinq premiers centimètres pour éviter la dispersion des invertébrés dans un volume trop important de sédiment. 
Tableau 2. Composition de la macrofaune benthique des lacs du Jura - variété générique aux différentes profondeurs - 14 lacs. D'après des données cumulées du Cemagref (1982-1987) et du Laboratoire d'Hydrobiologie (1979-1992) - 612 échantillons - ( ) : faune associée aux sédiments meubles.

Table 2. Composition of the benthic macrofauna of the lakes of the Jura. Generic variety at different depths. 14 lakes. From cumulated results of Cemagref (1982-1987) and Laboratoire d'Hydrobiologie (1979-1992). 612 samples. ( ) : soft-sediment related fauna.

\begin{tabular}{|c|c|c|c|c|c|c|c|c|c|c|c|c|c|}
\hline$Z$ (max) & Plécoptères & Odonates & Trichopteres & Ephóméroptères & Mégaloptères & Coleoptères & Diptères & Crustaces & Bivalves & Gasteropodes & Achètes & Otigochètes & $\Sigma$ \\
\hline$<2,5$ & 3 & $\begin{array}{l}11 \\
\text { (5) }\end{array}$ & $\begin{array}{c}21 \\
(11)\end{array}$ & $\begin{array}{l}10 \\
\text { (8) }\end{array}$ & 1 & 24 & 57 & 2 & 4 & 14 & 5 & 15 & $\begin{array}{c}167 \\
(118)\end{array}$ \\
\hline-5 & & & 6 & 6 & 1 & 4 & 38 & 2 & 3 & 6 & 2 & 17 & 88 \\
\hline$\cdot 10$ & & & 4 & 5 & 1 & 2 & 33 & 2 & 2 & 3 & & 11 & 63 \\
\hline .15 & & & 2 & & 1 & 2 & 22 & 1 & 2 & 2 & & 8 & 40 \\
\hline-20 & & & 1 & & 1 & 1 & 16 & & 1 & 1 & & 7 & 28 \\
\hline .25 & & & & & & 1 & 8 & & 1 & & & 6 & 16 \\
\hline 30 & & & & & & 1 & 7 & & 1 & & & 4 & 13 \\
\hline .35 & & & & & - & 1 & 4 & & 1 & & & 3 & 9 \\
\hline-40 & & & & & & & 3 & & & & & 2 & 5 \\
\hline
\end{tabular}

Les parties latérales ont été lestées afin d'assurer la pénétration dans les substrats compacts ; un limitateur de pénétration a été placé à l'intérieur du compartiment de récolte à une distance correspondant à un enfoncement de $5 \mathrm{~cm}$ pour un substrat compact, à faible teneur en eau, comme la craie lacustre, en zone littorale du lac de Clairvaux.

Les surcharges, de différents poids, sont ajustées à la fluidité du sédiment, de façon à obtenir au minimum $1 \mathrm{dm}^{3}$ de sédiment.

Le sédiment prélevé ( 2 bennes, $S \simeq 0,04 \mathrm{~m}^{2}, \mathrm{v} \geqslant 2 \mathrm{dm}^{3}$ ) est tamisé sur place dans un filet de $250{ }_{\mu} \mathrm{m}$ de vide de maille. Pour des sédiments très organiques, riches en complexes colloïdaux (lac de Bonlieu), une tamiseuse vibrante a été utilisée.

Afin d'éviter la formation d'acide formique, susceptible d'attaquer la structure calcaire des coquilles des Pisidies, le reliquat est fixé par une solution formolée tamponnée à l'aide de carbonate de calcium à saturation ( 25 $\mathrm{cm}^{3}$ de formol $5 \%$ tamponné par échantillon). L'échantillon est placé dans un sachet en matière plastique fermé sans emprisonner d'air. Les échantillons sont transportés en glacière puis conservés au réfrigérateur.

Les essais préliminaires ont confirmé que :

- le prélèvement des 5 premiers centimètres de sédiment suffit à récolter au moins $90 \%$ du macrobenthos (Giani \& Lucas 1974, Milbrink 1978),

- les rendements de capture du carottier (type Rofes, Savary 1981) utilisé précédemment (Verneaux et al. op. cit.) et de la benne modifiée sont équivalents (13-14 genres $/ 0,1 \mathrm{~m}^{2}$ au lac de l'Abbaye à $-2,5 \mathrm{~m}$ ),

- une variété générique similaire est obtenue pour 2 prélèvements par station avec la benne et pour 4 avec le carottier (Tableau 3).
Tableau 3. Accroissement de la variété générique en fonction du nombre de prélèvements-lac de l'Abbaye, 10 stations à $-2,5 \mathrm{~m}, \mathrm{a}=$ aire prospectée, $\mathrm{g}=$ variété générique.

Table 3. Increasing of the generic variety in relation to the number of samples. Lake of the Abbaye, 10 stations at 2,5 m ; $\mathrm{a}$ : concerned area ; $\mathrm{g}$ : generic variety.

\begin{tabular}{ccccc}
\hline $\mathrm{n}$ & $\begin{array}{c}\mathrm{g} \\
\text { carottier }\end{array}$ & $\begin{array}{c}\mathrm{a} \\
\left(\mathrm{m}^{2}\right)\end{array}$ & $\begin{array}{c}\mathrm{g} \\
\text { benne }\end{array}$ & $\begin{array}{c}\mathrm{a} \\
\left(\mathrm{m}^{2}\right)\end{array}$ \\
\hline 1 & 3 & 0.012 & 7 & 0.021 \\
2 & 4 & 0.024 & 8 & 0.044 \\
3 & 5 & 0.036 & 10 & 0.063 \\
4 & 8 & 0.048 & 12 & 0.084 \\
5 & 10 & 0.060 & 13 & 0.105 \\
6 & 10 & 0.072 & 19 & 0.136 \\
7 & 11 & 0.084 & 23 & 0.147 \\
8 & 13 & 0.096 & 24 & 0.168 \\
9 & 14 & 0.108 & 24 & 0.189 \\
10 & 15 & 0.120 & 25 & 0.210 \\
\hline
\end{tabular}

\section{Protocole d'échantillonnage}

\subsection{Période d'échantillonnage et unités taxonomiques}

$\mathrm{Si}$, comme dans la plupart des systèmes aquatiques d'Europe tempérée, les températures estivales (juillet-août) sont favorables aux émergences ima- 
ginales du plus grand nombre d'espèces, on observe que, dans les lacs jurassiens, situés entre 400 et $1000 \mathrm{~m}$ d'altitude, les premières éclosions sont plus précoces que dans les lacs pyrénéens étudiés par Laville (1971) et localisés au-dessus de $2000 \mathrm{~m}$.

Dans les lacs étudiés, suivant l'altitude et les années, le dégel s'opère au cours des mois de mars et d'avril, plus rarement en fin février ou au début du mois de mai.

Les émergences précoces, correspondant à l'atteinte d'une température de 7 à $8^{\circ} \mathrm{C}$, surviennent au cours de la période d'isothermie qui suit le dégel. Comme dans le lac Esrom (Jónasson 1961), cette période peut être repérée par les premières éclosions de Chironomus anthracinus Zetterstedt correspondant à l'atteinte de cette température et succédant aux premières éclosions de certains Tanytarsini comme Tanytarsus miriforceps Kieffer et Tanytarsus niger Andersen (07/04, lac de l'Abbaye, obs. pers.). Les campagnes d'échantillonnage ont été effectuées peu après le dégel de la surface du lac, au début de la phase isothermique dont la durée est de l'ordre de 15 jours.

L'impossibilité de fonder sur la détermination des espèces un protocole susceptible d'application simultanée à plusieurs lacs et qui prenne en compte l'ensemble du macrobenthos, conduit à se référer à la variété générique sauf pour les Cératopogonidés, les Hydracariens, les Nématodes et les Oligochètes pour lesquels on a distingué 3 groupes : Oligochètes autres que les Tubifidae, Tubificidae sans soies capillaires et Tubificidae avec soies capillaires.

\subsection{Nombre d'isobathes prospectées}

La régression de la faune, surtout de sa variété, avec la profondeur, phénomène général, constitue un élément essentiel de l'analyse comparée des systèmes. Les résultats des essais effectués en avril 1991 sur 3 lacs avec des sédiments nettement différents (Verneaux et al. 1987 et Tableau 1) sont portés sur la figure 1.

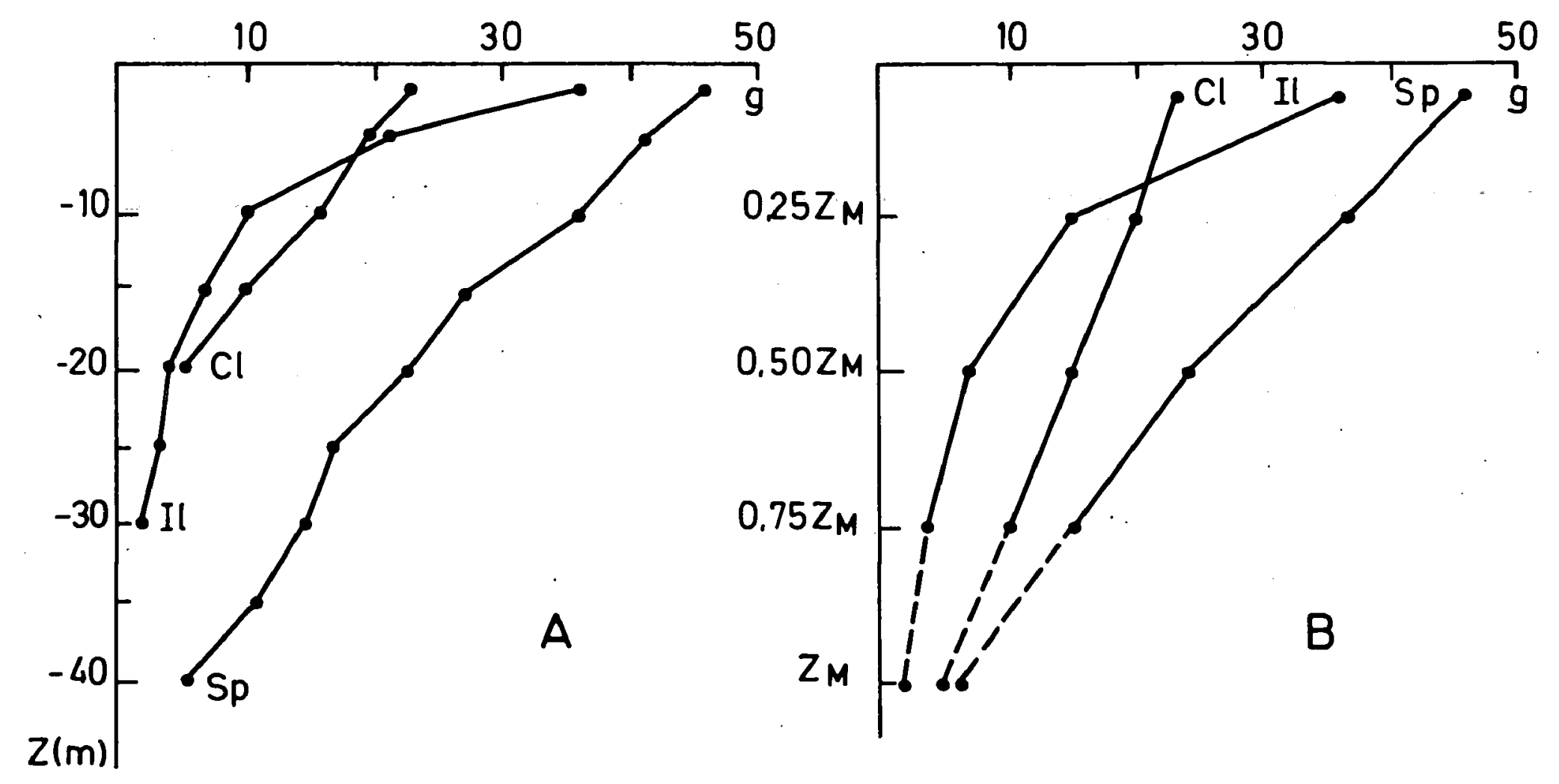

Fig. 1. Distribution du nombre de genres en fonction de la profondeur - 3 lacs jurassiens - 560 échantillons - A : profondeurs absolues, $\mathrm{B}$ : profondeurs relatives. $\mathrm{Cl}$ : Clairvaux, Il : Ilay, Sp : St-Point. Z(m) : profondeurs en mètres. ZM : profondeurs maximales.

Fig. 1. Distribution of the number of genera in relation to the depth. 3 lakes of the Jura. 560 samples - A : absolute depth, B : relative depth. $\mathrm{Cl}$ : Clairvaux, Il : llay, Sp : St-Point. $\mathrm{Z}(\mathrm{m})$ : depths in metres. ZM : maximum depths. 
Le nombre d'échantillons par isobathe prospectée est : 10 pour le lac de Clairvaux, 15 pour le lac d'Ilay, 20 pour le lac de St-Point.

Les courbes de distribution sont du même type que celles reproduites par Brinkhurst (1974) pour 7 lacs différents, à partir de la variété spécifique.

A la profondeur maximale des lacs, la variété de la macrofaune, réduite à quelques genres, ne permet qu'une faible discrimination des systèmes. Cette tendance à l'annulation de la variété à la profondeur maximale de la cuvette, quelle que soit cette dernière, conduit à fonder la comparaison des systèmes à profondeurs relatives égales. Sur la figure 1B, les courbes de distribution de la variété générique sont établies à partir des relevés effectués aux profondeurs de 1 à $2 \mathrm{~m}$ puis de $0,25,0,50$ et $0,75 \mathrm{Zmax}$.

La prise en compte de ces profondeurs relatives fournit des images fidèles et comparables de la distribution de la macrofaune benthique.

Les déficits génériques rapides, dès les cinq premiers mètres, observés pour les lacs d'Ilay et de l'Abbaye sont conservés et la différence entre les pentes des régressions faunistiques obtenues pour les lacs de St.Point et de Clairvaux met en évidence le fait que, dans les conditions favorables d'une régression de type linéaire, le déficit taxonomique relatif est d'autant plus grand (pente plus faible) que la variété générique littorale est élevée. Cette donnée sera prise en compte au cours de l'analyse comparée des systèmes.

\subsection{Nombre nécessaire d'échantillons par isobathe}

- Variabilité de l'échantillon en fonction de la profondeur

Sur la figure 2 sont portées les valeurs du coefficient de variation $\mathrm{v}=\mathrm{S} / \overline{\mathrm{x}}$, calculées pour la variété générique. L'inégalité du nombre d'échantillons effectués aux différentes profondeurs conduit à calculer un coefficient moyen de variation à partir des coefficients $\mathrm{v}$ calculés lac par lac à nombre constant de prélèvements ( 7 lacs, 9 profondeurs, 10 échantillons par profondeur et par lac).

La variabilité, forte en zone littorale, diminue rapidement, dès $-5 \mathrm{~m}$, conjointement à la variété faunistique, puis se stabilise. On note cependant une augmentation de la variabilité de la variété générique en zone très profonde où la pauvreté de la faune entraîne parfois des valeurs nulles. Cette observation conduit à exclure du protocole la prospection des profondeurs supérieures à $0.75 \mathrm{Zmax}$.

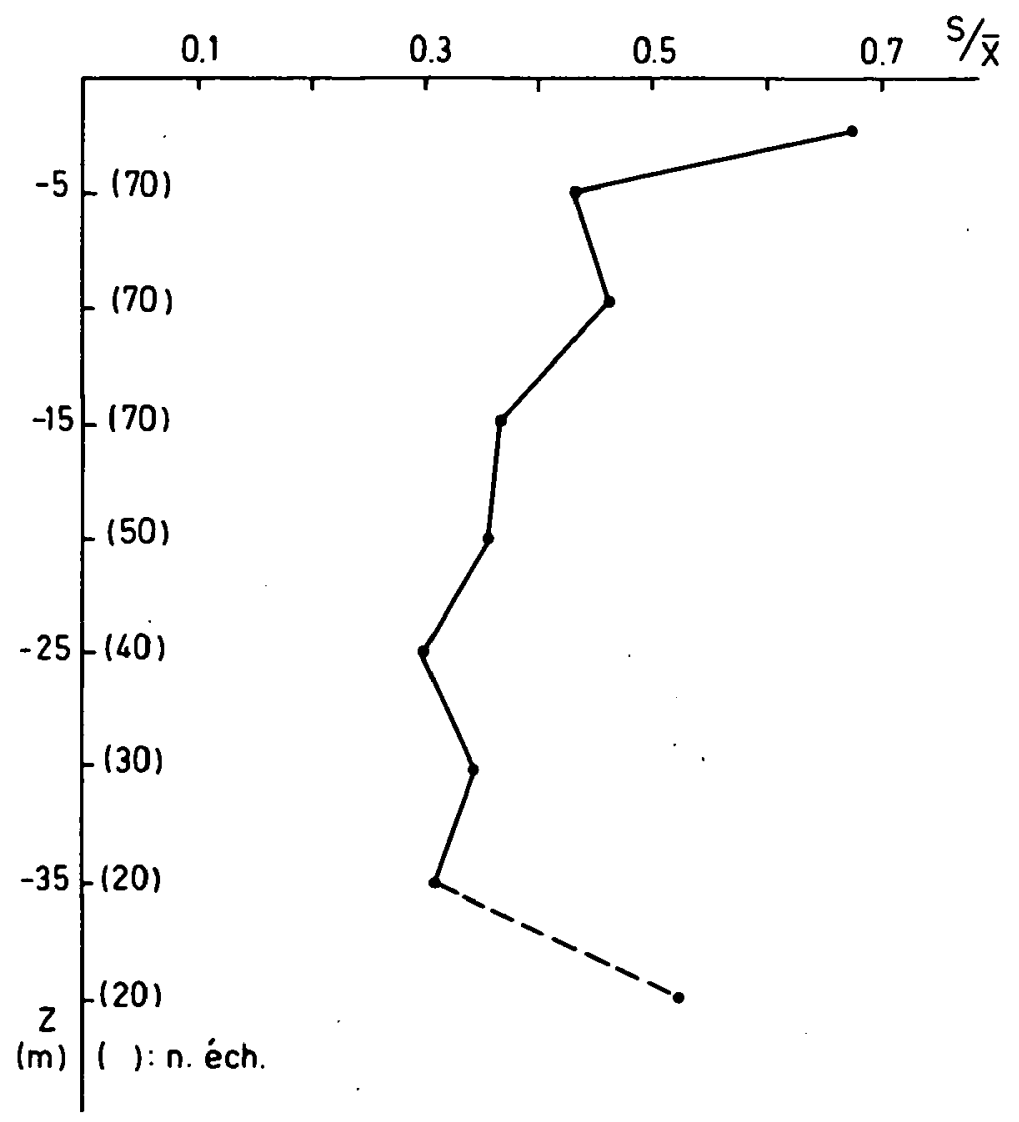

Fig. 2. Variabilité moyenne de la variété générique en fonction de la profondeur - 7 lacs jurassiens - 420 échantillons.

Fig. 2. Variability of the mean generic variety in relation ot the depth. 7 lakes of the Jura. 420 samples.

- Relation entre le nombre d'échantillons nécessaires et la longueur des isobathes

La méthode du cumul des taxons nouveaux (Gaufin et al. 1956) a été appliquée à la variété générique, l'atteinte du pallier (stabilisation) étant fixée à $90 \%$ de la variété générique totale.

Les calculs ont été effectués, pour les lacs de l'Abbaye, Châlain, Clairvaux, Ilay, Maclu, Remoray et St-Point, à partir des échantillons prélevés en avril 1989 et 1990 aux profondeurs relatives $Z_{0}, Z_{1}, Z_{2}$ et $Z_{3}$ précédemment définies, après vérification que le nombre de relevés par isobathe était au moins égal à 1.5 fois la valeur $\mathbf{n}$ obtenue par la méthode des taxons cumulés.

La figure 3 présente les variations du nombre $n$ d'échantillons à effectuer en fonction de la longueur $\mathrm{L}$ des isobathes. La variété générique et sa variabilité, étant toujours plus élevées en zone eulittorale (Figs 1,2 ), la relation entre $n$ et $L$ s'opère à deux niveaux, lorsque l'on joint les valeurs maximales de $n$ obtenues en $Z_{0}$ et aux profondeurs relatives $Z_{1}$, $Z_{2}$ et $Z_{3}$. 


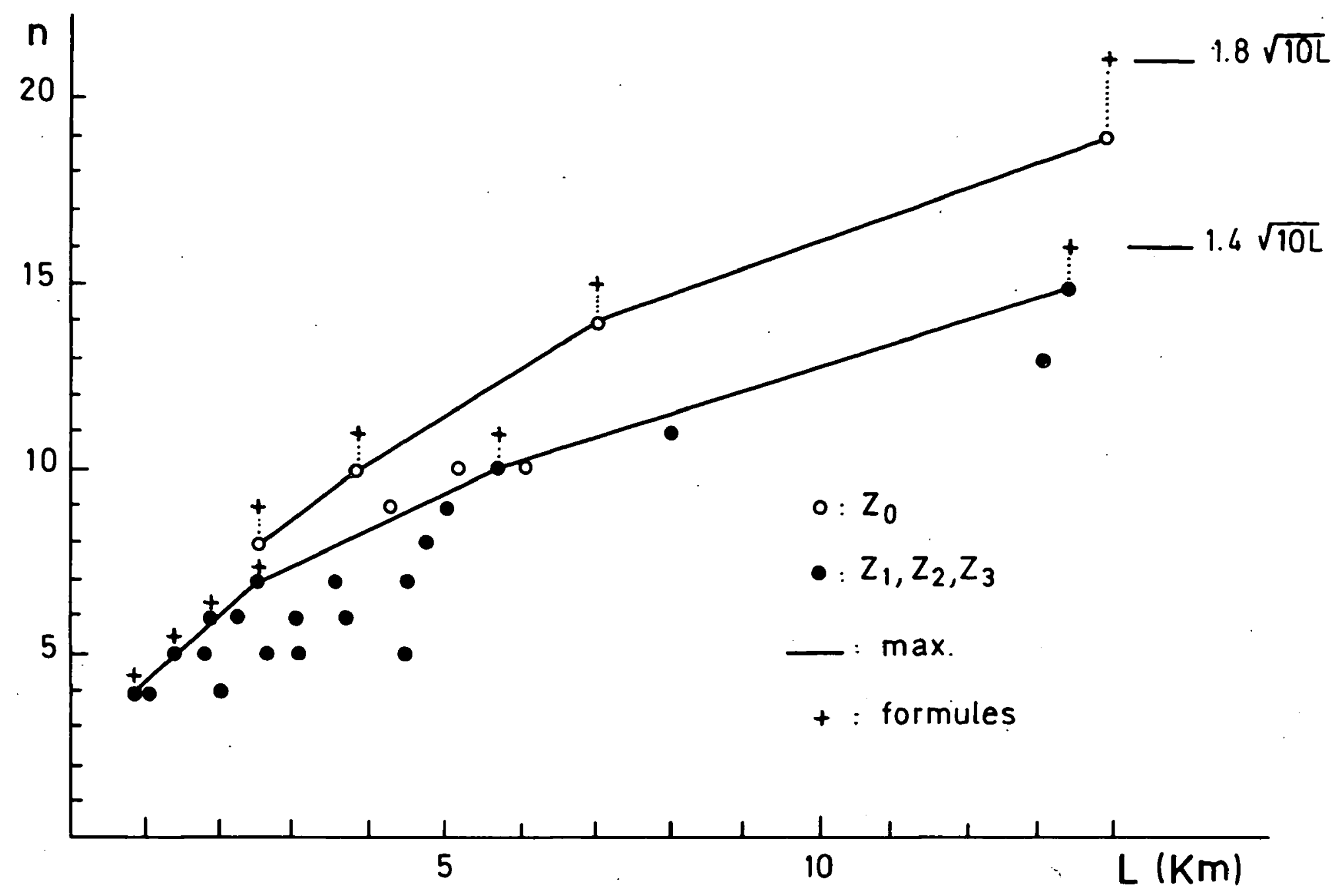

Fig. 3. Nombre nécessaire d'échantillons en relation avec la longueur des isobathes - 7 lacs - 452 échantillons.

Fig. 3. Required number of samples according to the length of the isobaths. 7 lakes - 452 samples.

Etant donné que les valeurs expérimentales obtenues ne constituent que des repères, nous avons choisi de retenir non les équations des courbes obtenues, sans réelle valeur statistique, mais deux relations $\mathrm{n}=\mathrm{f}(\mathrm{L})$ simples, permettant de définir rapidement $\mathbf{n}$ à partir des cartes bathymétriques.

Les valeurs théoriques portées sur la figure 3 : $\mathrm{n}=1.8 \sqrt{10 \mathrm{~L}}$ pour la profondeur $\mathrm{Z}_{0}$ et $\mathrm{n}=$ $1.4 \sqrt{10 \mathrm{~L}}$ pour les profondeurs relatives, sont très voisines des valeurs expérimentales et légèrement supérieures à ces dernières (marges de sécurité).

Les $\mathbf{n}$ stations sont, autant que possible, réparties de façon équidistante sur chaque isobathe et, pour les périmètres inférieurs à $0.78 \mathrm{~km}$, le nombre minimal de stations est fixé à 4 .

- Remarque sur la densité globale
Tableau 4. Densité (ind. $/ 0,04 \mathrm{~m}^{2}$ ) du macrobenthos en 3 profondeurs au lac de St.Point ( 30 prélèvements par profondeur) et nombre d'échantillons nécessaires.

Table 4. Macrobenthos density (ind. per $0,04 \mathrm{~m}^{2}$ ) at 3 depths in the lake St Point, (30 samples at each depth) and required number of samples.

\begin{tabular}{llll}
$\mathrm{Zm}$ & $-2,5$ & -10 & -30 \\
\hline $\bar{x}$ & 62,4 & 73,2 & 23,0 \\
$\mathrm{~S}^{2}$ & 4239 & 5804 & 541 \\
$\mathrm{I}=\mathrm{S}^{2} \overline{\mathrm{x}}$ & 62,6 & 79,2 & 23,5 \\
$\mathrm{n}=\mathrm{S}^{2} / \mathrm{D}^{2} \cdot \overline{\mathrm{x}}^{2}$ & 27 & 27 & 25 \\
$\mathrm{n}^{\prime}=$ moy. progr. & 23 & 21 & 17 \\
\hline
\end{tabular}


Les nombres minimaux d'échantillons calculés d'après les valeurs de densité globale sont plus élevés que ceux calculés d'après la variété générique. L'application de la formule d'Elliott (1977) donne des valeurs toujours plus élevées que celles obtenues par la méthode des moyennes progressives (Ricou 1967), en fixant dans les deux cas une tolérance de $20 \%$ sur la moyenne. Dans le cas présenté du lac de St-Point, le nombre nécessaire de prélèvements est de l'ordre de 25 en zone littorale et de 20 en zone profonde, valeurs supérieures mais cependant comparables à celles obtenues pour la variété générique, considérée comme critère principal de l'analyse comparative.

Toutefois, cette relative stabilisation de la densité faunistique globale résulte des compensations entre les abondances des différents genres. Si elle permet une comparaison globale des systèmes, elle n'implique pas l'obtention de densités stabilisées pour les différents genres.

\subsection{Vérification et tableau d'échantillonnage comparatif}

Pour les Diptères Chironomidés du lac de Clairvaux, il a été vérifié que l'application du protocole proposé n'amputait pas le bilan relatif à la variété générique (Tableau 5).
Les résultats de Wasson (1984) concernent 3 campagnes d'échantillonnage (avril, juillet, octobre) avec chacune 3 prélèvements par profondeur. Nos résultats proviennent d'une seule campagne d'échantillonnage (avril 1990) avec 24, 14, 14 et 12 prélèvements régulièrement répartis sur les 4 isobathes considérées.

Sur les 29 genres recensés, 21 figurent parmi les 22 genres répertoriés par Wasson (op. cit.).

Tableau 5. Comparaison des variétés génériques des larves de Diptères Chironomidés prélevées au lac de Clairvaux - Pour chaque profondeur, colonne de gauche : d'après Wasson, 1984, colonne de droite : d'après le protocole proposé appliqué en 1990.

Table 5. Comparison between generic variety of the larvae of Chironomidae (Diptera) sampled at each depth in the lake Clairvaux, from Wasson 1984 (left column) and from the proposed sampling method used in 1990 (right column).

\begin{tabular}{lllllllll}
\hline Taxons $Z(\mathrm{~m})$ & -2 & \multicolumn{1}{c}{-5} & & -10 & -20 \\
\hline Orthocladiinae & 1 & 4 & 1 & 3 & 2 & 3 & - & - \\
Tanypodinae & 2 & 2 & 2 & 2 & 1 & 1 & 1 & 1 \\
Tanytarsini & 4 & 5 & 4 & 4 & 3 & 3 & 1 & 1 \\
Chironomini & 8 & 7 & 4 & 8 & 10 & 9 & 3 & 4 \\
\hline
\end{tabular}

Dans les conditions de prélèvement précédemment définies, le tableau 6 récapitule le protocole d'échantillonnage appliqué à l'étude comparative des 9 lacs de la France-Comté jurassienne.

Tableau 6. Protocole d'échantillonnage utilisé pour l'étude comparative de la macrofaune benthique des sédiments lacustres - Z : profondeur d'échantillonnage - L : longueur de l'isobathe considérée - $n$ : nombre d'échantillons par isobathe $(n=k \sqrt{10} \mathrm{~L})$.

Table 6. Sampling method used for the comparative survey of the benthic macrofaune of the lake sediments $-Z$ : depth of sampling - L : length of the particular isobath (in $\mathrm{km})-\mathrm{n}:$ number of samples at each isobath $(n=k \sqrt{10 \mathrm{~L}})$.

\begin{tabular}{|c|c|c|c|c|c|c|c|c|c|}
\hline & $A b$ & Bo & Ch & $\mathrm{Cl}$ & II & $\mathrm{Ma}$ & $\mathrm{Na}$ & $\mathrm{Re}{ }^{\circ}$ & Sp \\
\hline $\mathrm{Zo}(\mathrm{m})$ & $1-2$ & $1 \cdot 2$ & $1-2$ & $1-2$ & $1 \cdot 2$ & $1-2$ & $1-2$ & $1 \cdot 2$ & $1-2$ \\
\hline $\mathrm{Z1}(\mathrm{m})$ & 4,5 & 3,8 & 8 & 5 & 8 & 6,3 & 10 & 5 & 10 \\
\hline $\mathrm{Z2}(\mathrm{m})$ & 9 & 7,5 & 15 & 10 & 16 & 12,5 & 20 & 13 & 20 \\
\hline $23(\mathrm{~m})$ & 13,5 & 11,3 & 24 & 15 & 24 & 18,8 & 30 & 20 & 30 \\
\hline Lo (km) & 6 & 1,8 & 7,4 & 4,3 & 5,2 & 2,5 & 2,9 & 3,8 & $13 ; 8$ \\
\hline L1 (km) & 4,5 & 1,7 & 5,7 & 2,5 & 4,8 & 1,9 & 7 & 3,5 & 13,4 \\
\hline L2 (km) & 3 & 1,6 & 5 & 2,3 & 3,6 & 1,4 & 2 & 3 & 13 \\
\hline L3 (km) & 1 & 1,4 & 4,5 & 1,7 & 2 & 0,8 & 1 & 2,6 & 8 \\
\hline no & 14 & 8 & 15 & 12 & 13 & 9 & 10 & 11 & 21 \\
\hline$n 1$ & 9 & 8 & 11 & 7 & 10 & 6 & 7 & 8 & 16 \\
\hline $\mathrm{n} 2$ & 8 & 6 & 10 & 7 & 8 & 5 & 6 & 8 & 16 \\
\hline n3 & 4 & 5 & 9 & 6 & 6 & 4 & 4 & 7 & 13 \\
\hline$\Sigma$ & 35 & 25 & 45 & 32 & 37 & 24 & 27 & 34 & 66 \\
\hline
\end{tabular}




\section{Résultats}

\subsection{Répertoire des taxons génériques}

650 prélèvements ( 325 échantillons) ont été réalisés en avril-mai 1989-90-91. 104 taxons dont 98 genres ont été identifiés sur les 14205 individus dénombrés, sans recours au sous-échantillonnage.

La nature et la distribution générale des taxons figurent dans le tableau 7 où sont indiquées : la fréquence relative aux lacs $\left(\mathrm{N}_{1}\right)$ et aux stations $\left(\mathrm{N}_{2}\right)$, la densité moyenne rapportée à $0,1 \mathrm{~m}^{2}$ de surface sédimentaire, ainsi que la densité (\%) de chaque taxon par rapport à la densité globale.

Dans les lacs étudiés et dans les conditions d'échantillonnage données, les Diptères Chironomidés dominent largement la faune des sédiments. Le fait a été mentionné par les nombreux auteurs ayant étudié des systèmes géographiquement et morphologiquement très différents (Oliver 1960, Slack 1965, Bonomi \& Ruggiu 1966, Bagge 1969, Macan 1970, Wiederholm \& Eriksson 1977, Prat 1978).

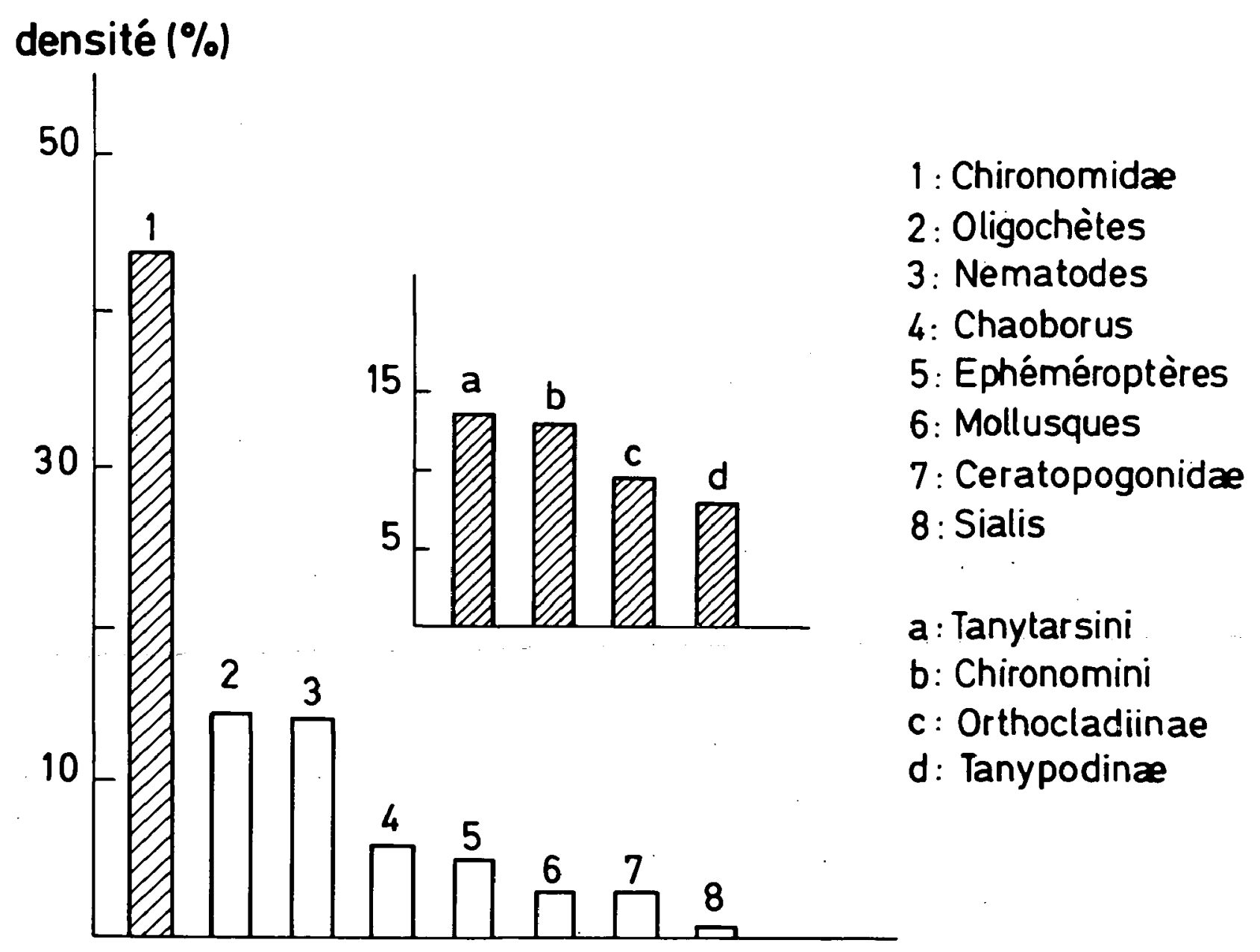

Fig. 4. Composition de l'échantillon global - Macrobenthos des sédiments de 9 lacs du Jura.

Fig. 4. Total sample composition. Sediment macrobenthos in 9 lakes of the Jura.

On note cependant une grande variabilité de la participation à la densité des taxons comme les Amphiphodes ou les Bivalves. Les lacs étudiés entrent tous dans la catégorie des lacs à Chaoborus (lacs à Corethra de Alm 1922 in Brinkhurst 1974) mais les Tanytarsini, comme dans le lac Léman (Juget 1958), dominent la faune diptérienne. Parmi les insectes, autres que les Diptères, les Odonates et les Trichoptères sont limités à la zone littorale ( $\mathrm{Z}$ $<10 \mathrm{~m}$ ). Brundin (1949) mentionne toutefois l'aug- mentation de l'amplitude bathymétrique de ces derniers, des systèmes « polyhumiques » aux systèmes « oligohumiques » jusqu'à des profondeurs de $20 \mathrm{~m}$.

Chez les Ephéméroptères, seuls les genres Ephemera et surtout Caenis ont été recensés en zone profonde ainsi que les genres Sialis (Mégaloptères) et Elmis (Coléoptères). Ce dernier genre n'a été récolté à $-38 \mathrm{~m}$ que dans le lac de St-Point, traversé par le Doubs dont l'influence est notoire sur la répartition des sédiments (Verneaux et al. 1988). 
Tableau 7. Répertoire des taxons (genres) répertoriés à l'aide du protocole proposé dans les sédiments de 9 lacs duJura. N1 : fréquence des taxons dans les lacs (\%). N2 : fréquence des taxons dans les stations (\%).

Table 7. List of taxa (genera) collected in the sediments of 9 lakes of the Jura using the proposed sampling method. N1 : frequency of the taxa in lakes $(\%)$. N2 : frequency of the taxa in stations $(\%)$.

\begin{tabular}{|c|c|c|c|c|c|}
\hline Taxons (genres) & Code & fréq & uence \% & $d$ & $\% \Sigma d$ \\
\hline & $N^{\circ}$ & $\begin{array}{l}\text { LACS } \\
\text { (N1) }\end{array}$ & $\begin{array}{l}\text { stations } \\
\text { (N2) }\end{array}$ & $0,1 \mathrm{~m} 2$ & \\
\hline ODONATES & 1 & 50 & 11 & & 0,71 \\
\hline Aeschna & & 22 & 5 & 0,15 & 0,07 \\
\hline Cordulia & & 11 & 2 & 0,30 & 0,13 \\
\hline Gomphus & & 11 & & 0,30 & 0,13 \\
\hline Platycnemis & & 22 & 5 & 0,45 & 0,80 \\
\hline Ophiogomphus & & 11 & 2 & 0,40 & 0,14 \\
\hline TRICHOPTERES & 2 & 100 & 52 & & 5,81 \\
\hline Agrypnia & & 33 & 8 & 0.40 & 0,18 \\
\hline Athripsodes & & 22 & 5 & 1,75 & 0.80 \\
\hline Beraeodes & & 11 & 5 & 0,35 & 0,16 \\
\hline Cyrnus & & 22 & 11 & 0,80 & 0,36 \\
\hline Ecnomus & & 44 & 13 & 0,92 & 0,41 \\
\hline Holocentropus & & 33 & 8 & 1,10 & 0,50 \\
\hline Limnephilini & & 22 & 5 & 0,25 & 0,11 \\
\hline Lype & & 11 & 2 & 2,00 & 0,90 \\
\hline Molanna & & 22 & 5 & 0,40 & 0,18 \\
\hline Mystacides & & 22 & 5 & 0,30 & 0,13 \\
\hline Oecetis & & 11 & 2 & 0,30 & 0,13 \\
\hline Oxyethira & & 11 & 2 & 0,50 & 0,13 \\
\hline Plectrocnemia & & 11 & 5 & 0,40 & 0,22 \\
\hline Polycentropus & & 11 & 2 & 0,20 & 0,18 \\
\hline Sericostoma & & 22 & 5 & 0,35 & 0,10 \\
\hline Setodes & & 22 & 5 & 1,40 & 0,16 \\
\hline Tinodes & & 11 & 2 & 0,30 & 0,63 \\
\hline & & & & & 0,13 \\
\hline EPHEMEROPTERES & 3 & & & & 4,58 \\
\hline Baetis & & 11 & 2 & 0,10 & 0,04 \\
\hline Caenis & 5 & 88 & 47 & 7,97 & 3,60 \\
\hline Ephemera & 4 & 66 & 25 & 1,65 & 0,74 \\
\hline Paraleptophlebia & & 11 & 2 & 0,35 & 0,16 \\
\hline Siphlonurus & & 11 & 2 & 0,10 & 0,04 \\
\hline COLEOPTERES & 6 & & & & 1,48 \\
\hline Donacia & & 11 & 2 & 1,80 & 0,81 \\
\hline Elmis & & 22 & 13 & 0,34 & 0,15 \\
\hline Enochrus & & 11 & 2 & 0,20 & 0,10 \\
\hline Haliplus & & 11 & 2 & 0,20 & 0,10 \\
\hline Platoumaris & & 11 & 2 & 0,70 & 0,32 \\
\hline MEGALOPTERES & & & & & 0,70 \\
\hline Sialis & 7 & 100 & 50 & 1,49 & 0,70 \\
\hline
\end{tabular}

\begin{tabular}{|c|c|c|c|c|c|}
\hline Taxons (genres) & Code & fréqu & ence $\%$ & $d$ & $\% \Sigma d$ \\
\hline & $N^{\circ}$ & $\begin{array}{l}\text { Lacs } \\
\text { (N1) }\end{array}$ & $\begin{array}{l}\text { stations } \\
\text { (N2) }\end{array}$ & $0,1 \mathrm{~m} 2$ & \\
\hline DIPTERES & & & & & 53,32 \\
\hline CERATOPOGONIDAE & 8 & 88 & 52 & 6,1 & 2,75 \\
\hline CHAOBORIDAE & & & . & & \\
\hline Chaoborus & 9 & 100 & 72 & 14,30 & 6,43 \\
\hline LIMONIIDAE & & & & & \\
\hline Helius & & 11 & 2 & 0,10 & 0,04 \\
\hline TABANIDAE & & & & & \\
\hline Tabanus & & 11 & 2 & 0,20 & 0,10 \\
\hline CHIRONOMIDAE & & & & & 44,00 \\
\hline${ }^{\circ}$ ORTHOCLADIINAE & & 88 & 55 & & 9,66 \\
\hline Cricotopus & 10 & 44 & 11 & 0,25 & 0,11 \\
\hline Epoicocladius & & 22 & 5 & 1,70 & 0,76 \\
\hline Eukiefferiella & & 22 & 5 & 0,35 & 0,16 \\
\hline Nanocladius & 11 & 22 & 14 & 0,50 & 0,22 \\
\hline Orthocladius & 12 & 33 & 8 & 5,10 & 2,30 \\
\hline Paracladius & 13 & 33 & 16 & 0,55 & 0,25 \\
\hline Psectrocladius & 14 & 88 & 22 & 8,30 & 3,73 \\
\hline Parakiefferiella & 15 & 22 & 8 & 5,33 & 2,13 \\
\hline 'PRODIAMESINAE & & & & & 0,10 \\
\hline Prodiamesa & & 11 & 2 & 0,20 & 0,10 \\
\hline -TANYPODINAE & & & & & 7,84 \\
\hline Ablabesmyia & 16 & 88 & 47 & 2,38 & 1,10 \\
\hline Apsectrotanypus & & 11 & 2 & 0,10 & 0,04 \\
\hline Clinotanypus & & 11 & 2 & 0,10 & 0,04 \\
\hline Labrundinia & & 11 & 2 & 0,30 & 0,13 \\
\hline Macropelopia & & 11 & 2 & 0,10 & 0,04 \\
\hline Procladius & 17 & 100 & 83 & 6,80 & 3,06 \\
\hline Tanypus & 18 & 44 & 25 & 1,54 & 0,70 \\
\hline Thienemannimyia & 19 & 22 & 11 & 3,07 & 1,38 \\
\hline Pentaneurini $x$ & & 22 & 16 & 1,81 & 0,81 \\
\hline Pentaneurini $y$ & 20 & 11 & 22 & 1,20 & 0,54 \\
\hline
\end{tabular}


Tableau 7. Suite.

\begin{tabular}{l} 
Taxons (genres) \\
\hline \\
\hline 'CHIRONOMINAE \\
'Tanytarsini \\
Cladotanytarsus \\
Constempellina \\
Micropsectra \\
Paratanytarsus \\
Rheotanytarsus \\
Stempellina \\
Stempellinella \\
Tanytarsus \\
'Chironomini \\
Chironomus \\
Cladopelma \\
Cryptochironomus \\
Cryptocladopelma \\
Cryptotendipes \\
Demicryptochironomus \\
Dicrotendipes \\
Einfeldia \\
Endochironomus \\
Glyptotendipes \\
Microtendipes \\
Pagastiella \\
Parachironomus \\
Paracladopelma \\
Paratendipes \\
Phaenopsectra \\
Polypedilum \\
Pseudochironomus \\
Tribelos \\
\end{tabular}

\section{MOLLUSQUES}

BIVALVES

Pisidium

Sphaerium

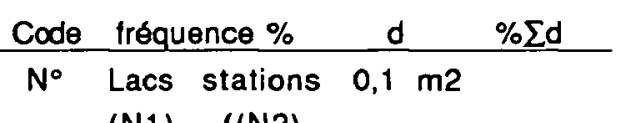

(N1) ((N2)

$\begin{array}{cccccl}21 & 100 & 83 & 9.30 & \mathbf{1 3 , 3 8} & \text { GASTEROPODES } \\ 22 & 33 & 8 & 0,40 & 0,18 & \text { Bithynia } \\ 23 & 33 & 8 & 1,27 & 0,57 & \text { Bythinella } \\ 24 & 100 & 56 & 1,69 & 0,76 & \text { Gyraulus } \\ 25 & 22 & 8 & 0.30 & 0,13 & \text { Lymnaea } \\ 26 & 11 & 8 & 0,46 & 0,21 & \text { Planorbis } \\ 27 & 33 & 14 & 0,54 & 0,24 & \text { Valvata }\end{array}$

$\begin{array}{llll}33 & 14 & 0,54 & 0,24\end{array}$

$28 \quad 100 \quad 86 \quad 15-60 \quad 7,82$

$\begin{array}{ccccc}30 & 100 & 83 & 4,21 & 2,12 \\ 29 & 100 & 47 & 1,11 & 0,50 \\ 31 & 44 & 25 & 1,66 & 0,75 \\ 32 & 33 & 11 & 0,15 & 0,07 \\ 33 & 33 & 11 & 0,77 & 0,35 \\ 34 & 66 & 28 & 0,32 & 0,14 \\ 35 & 88 & 44 & 2,19 & 0,98 \\ 36 & 100 & 77 & 4,61 & 2,07 \\ 37 & 55 & 25 & 1,48 & 0,57 \\ 38 & 55 & 19 & 0,47 & 0,21 \\ 39 & 55 & 33 & 0,92 & 0,42 \\ 40 & 44 & 16 & 1,57 & 0,71 \\ 41 & 55 & 28 & 0,60 & 0,27 \\ 42 & 44 & 28 & 0,39 & 0,17 \\ 43 & 66 & 36 & 0,53 & 0,30 \\ & 11 & 2 & 0,10 & 0,04 \\ 44 & 77 & 39 & 3,30 & 1,50 \\ 45 & 44 & 28 & 3,30 & 1,50 \\ 46 & 44 & 22 & 0,65 & 0,30\end{array}$

HYORACARIENS

CRUSTACES

Asellus

Gammarus

TRICLADES

Dugesia

\section{ACHETES}

Erpobdella

Glossiphonia

Helobdella

OLIGOCHETES

Autres que Tubificidae

Tubificidae

sans soies capillaires

avec soies capillaires

NEMATODES

$\begin{array}{ccccc}47 & 100 & 75 & 3,40 & 1,75 \\ & 22 & 22 & 0,50 & 0,22\end{array}$

Chez les Mollusques, seuls les genres Bithynia et surtout Pisidium ont été récoltés en zone profonde. Dans certains systèmes du type « à Pisidium » (de Valle 1927 in Brinkhurst 1969) ce mollusque constitue le genre dominant de la zone profonde. Les profondeurs maximum atteintes par ce genre dans plus de 30 lacs européens ont été recensées par Mouthon (1986).

\subsection{Analyse de la matrice générale : stations-taxons}

Une analyse factorielle des correspondances (Benzecri 1973) a été appliquée à l'analyse des proximités entre les lacs considérés aux 4 profondeurs rela- tives $Z_{0}, Z_{1}, Z_{2}$ et $Z_{3}(9 \times 4$ colonnes $)$ et les 54 taxons (lignes) tels qu'ils ont été présentés dans le répertoire (Tableau 7) après regroupement des genres à très faible fréquence et limités à la zone littorale, comme les Odonates et les Trichoptères.

Les analyses ont été pratiquées à partir des valeurs brutes de la densité de chaque taxon, pondérées par leurs densités moyenne et maximale. Ces différentes analyses donnent des résultats très voisins et le graphe de la figure 5 présente ceux d'une AFC globale effectuée à partir des pourcentages de densité de chaque taxon $\left(0 \leqslant \mathrm{~d}=\mathrm{di} / \mathrm{dM} .10^{2} \leqslant 100\right)$ dans les différents lacs, aux 4 profondeurs relatives. 


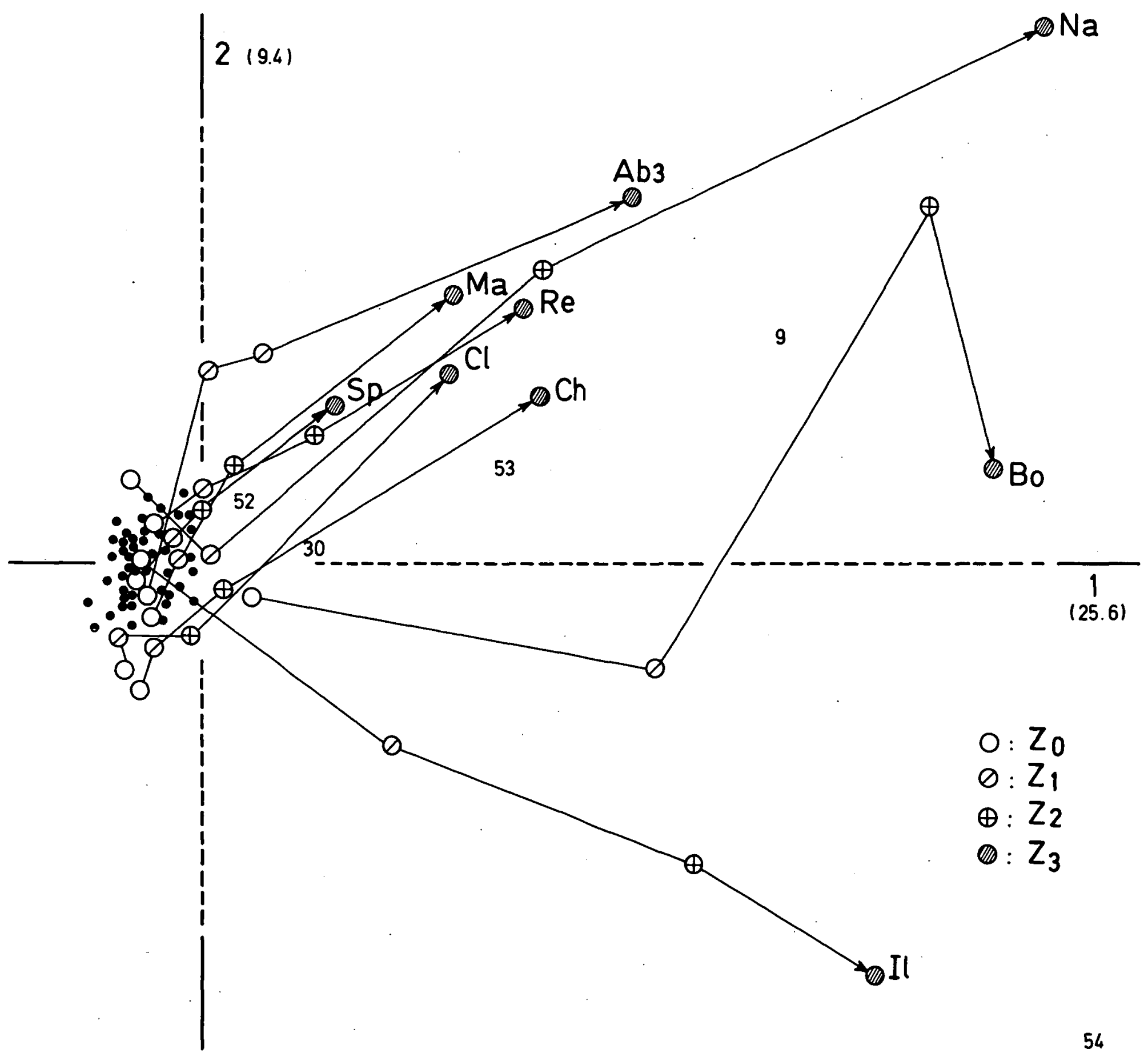

Fig. 5. Distribution des taxons et des stations dans le plan des 2 premiers axes d'une AFC globale. 54 taxons - 9 lacs - 36 stations 325 échantillons.

Fig. 5. Stations and taxa distribution in the first two axes system of a total factorial correspondence analysis. 54 taxa - 9 lakes - 36 stations - 325 samples.

Cinq taxons représentent $86 \%$ de la contribution totale au premier axe et, à l'exception des Nématodes $\left(\mathrm{N}_{2}=31 \%\right)$, ils possèdent tous une fréquence au moins égale à $70 \%$ des relevés (Tableau 1). Il s'agit de Chaoborus (9), des Tubificidae sans soies capillaires (52), de Chironomus (30) et des Tubificidae à soies capillaires (53) les plus tolérants (Lafont 1989).
La discrimination s'opérant secondairement sur le deuxième axe est surtout imputable aux Nématodes macroscopiques (54), uniquement abondants dans le lac d'Ilay. La plupart des Nématodes n'étant pas retenus par la maille du tamis utilisé $(250 \mu \mathrm{m})$ la différenciation obtenue à l'aide de cette classe n'est pas retenue. 
Ces taxons discriminants et les stations se succèdent suivant le premier axe dans l'ordre de profondeur croissante et de variété taxonomique décroissante. Cet axe exprime essentiellement la variation des 2 paramètres d'analyse des peuplements : variété et densité en fonction de la modification des paramètres physiques et chimiques de l'eau et du sédiment qui s'effectue selon la profondeur.

La figure 6 représente les distances à l'origine des axes des projections des stations sur le premier axe (coord. F1).

On observe l'accroissement de la différenciation des lacs selon l'amplitude du déficit taxonomique (générique) relevé aux profondeurs relatives successives. Il en résulte un classement des lacs dans un ordre de qualité biologique décroissante de St-Point à Narlay.

\subsection{Variété générique totale et densité globale moyenne}

Un classement des lacs est obtenu en fonction de la variété générique totale et de l'abondance globale moyenne de la macrofaune des sédiments (Fig. 7).

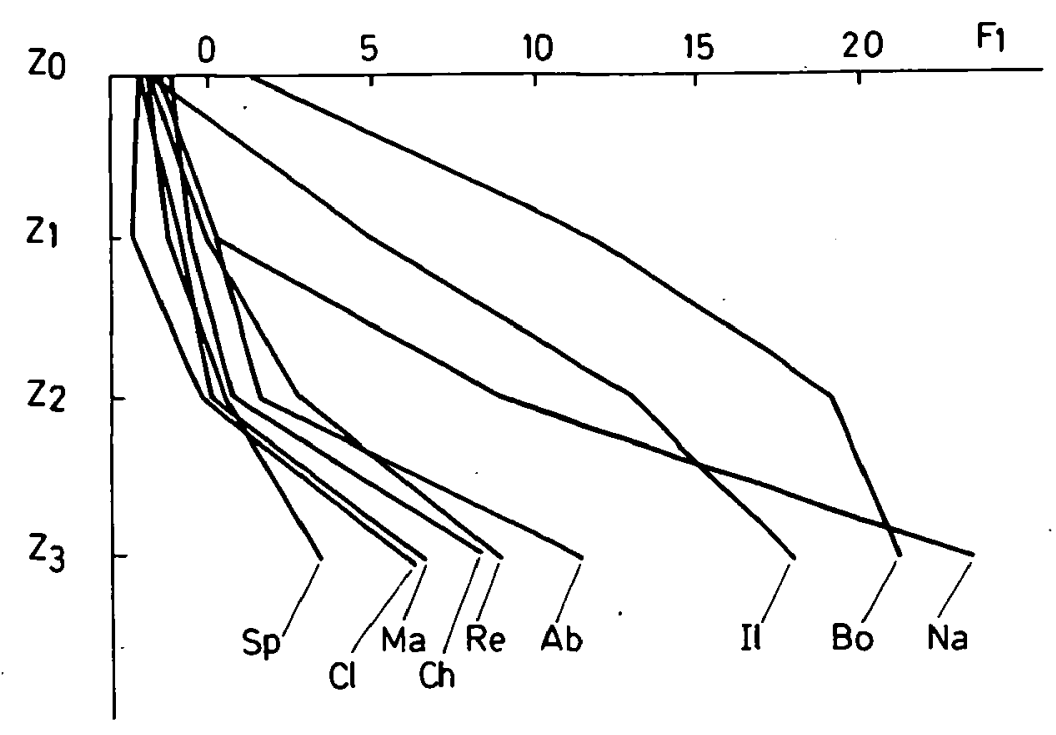

Fig. 6. Classement des lacs obtenu par la projection des stations sur le premier axe de l'AFC aux profondeurs relatives successives. En abscisse : distances à l'origine des axes.

Fig. 6. Classification of lakes obtained with projecting the stations on the first axis of the factorial correspondence analysis at successive relative depth. On abscissa : distances from - axis origin.

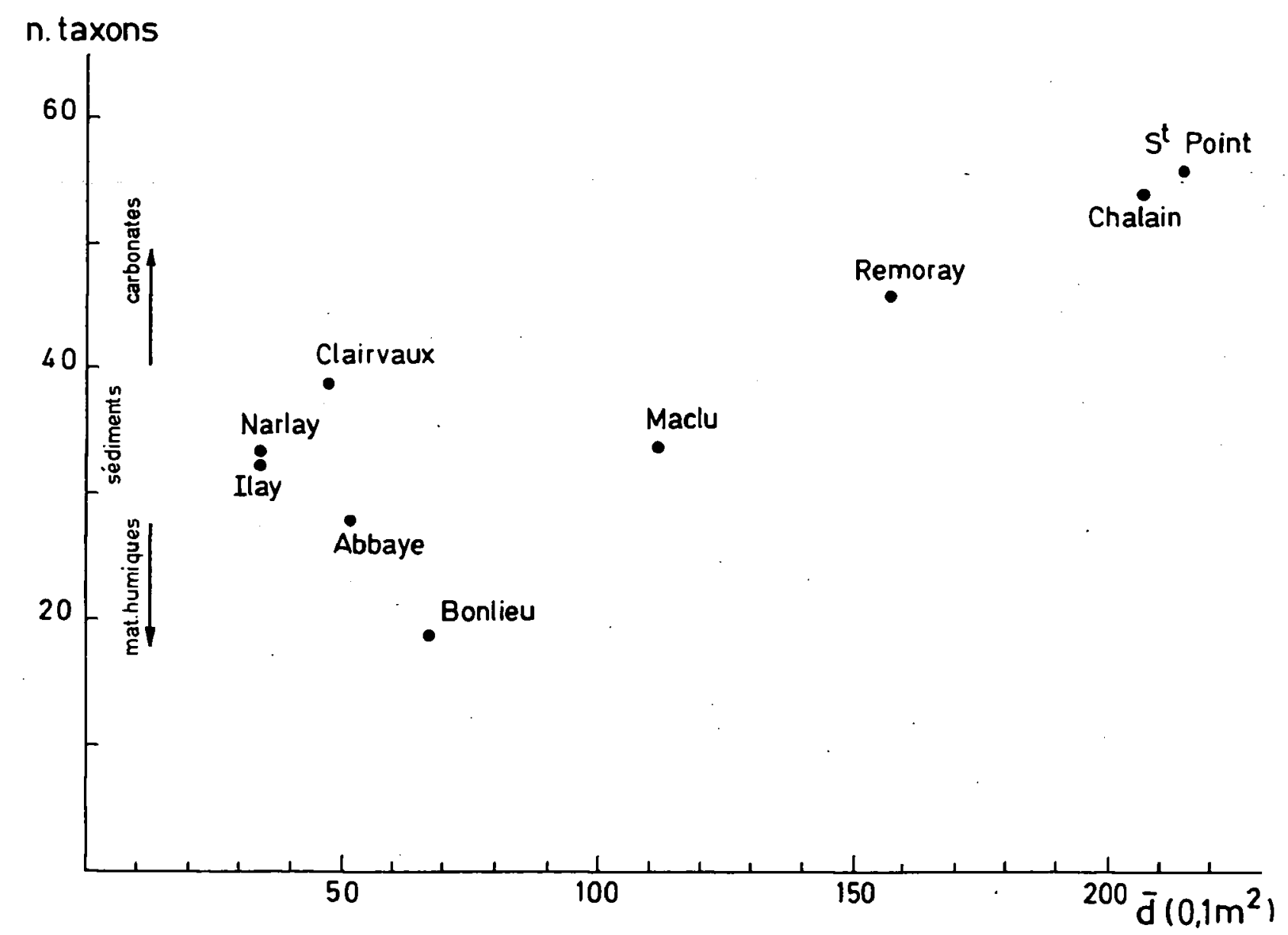

Fig. 7. Classement des lacs en fonction de la variété taxonomique totale et de la densité moyenne globale du macrobenthos.

Fig. 7. Classification of lakes according to the total taxonomic variety and the mean total density of the macrobenthos. 
Du lac de Bonlieu, aux sédiments riches en matière organique au lac de St-Point à faible teneur en matière organique résiduelle (Tableau 1), la répartition s'effectue suivant une droite joignant les combinaisons minimale et maximale du couple variétédensité. Dans le groupe des lacs à faible densité faunistique une différenciation s'établit de Bonlieu à Clairvaux d'après la variété portée en ordonnée. Toutefois, le couple v-d ne permet pas de différencier des lacs comme Clairvaux, Narlay et Ilay aux caractéristiques écologiques nettement différentes, mais qui se trouvent regroupés dans le système d'axes considéré. Ainsi, le lac de Clairvaux, peu productif et aux sédiments très pauvres en matière organique (MO $<5 \%$ ) demeure proche du lac de Narlay, pollué par des eaux résiduelles karstiques profondes, très désoxygéné et accumulant la matière protéique à $\mathrm{C} / \mathrm{N}$ bas, due à des accumulations de filaments d'Oscillatoria rubescens (Verneaux et al. 1991).

\subsection{Variation de la densité et de la variété taxono- mique en fonction de la profondeur relative}

\section{La densité}

A l'exception du lac de Bonlieu, le moins biogène des lacs étudiés, la densité décroît avec la profondeur
(Fig. 8A). Cette diminution est observée par plusieurs auteurs. Toutefois, il existe une grande disparité entre les différentes études et il s'avère difficile d'effectuer une étude bibliographique comparative du fait de la variété des protocoles d'échantillonnage utilisés. Si l'on exclut du décompte, les Nématodes, les Oligochètes et le genre Chaoborus, responsables de certaines augmentations de la densité avec la profondeur, le phénomène observé se trouve accentué et prend un caractère général, dans le cas des lacs étudiés (Fig. 8B).

L'augmentation de la densité des Oligochètes et des Nématodes avec la profondeur est signalée par Rawson (1953) jusqu'à des profondeurs supérieures à $500 \mathrm{~m}$, mais elle ne constitue cependant pas une constante puisque, selon Bagge (1969), dans certains lacs de Finlande, la densité de ces deux groupes suit la tendance inverse. Ces observations conduisent à ne prendre en compte la densité qu'au niveau du bilan qui sera proposé pour la zone littorale (Zo, Fig. 11).

\section{La variété générique}

La figure 9 met en évidence la tendance à la linéarité des régressions de la variété générique en fonction de la profondeur relative, observée pour les lacs les plus biogènes (St-Point, Châlain, Fig. 7).

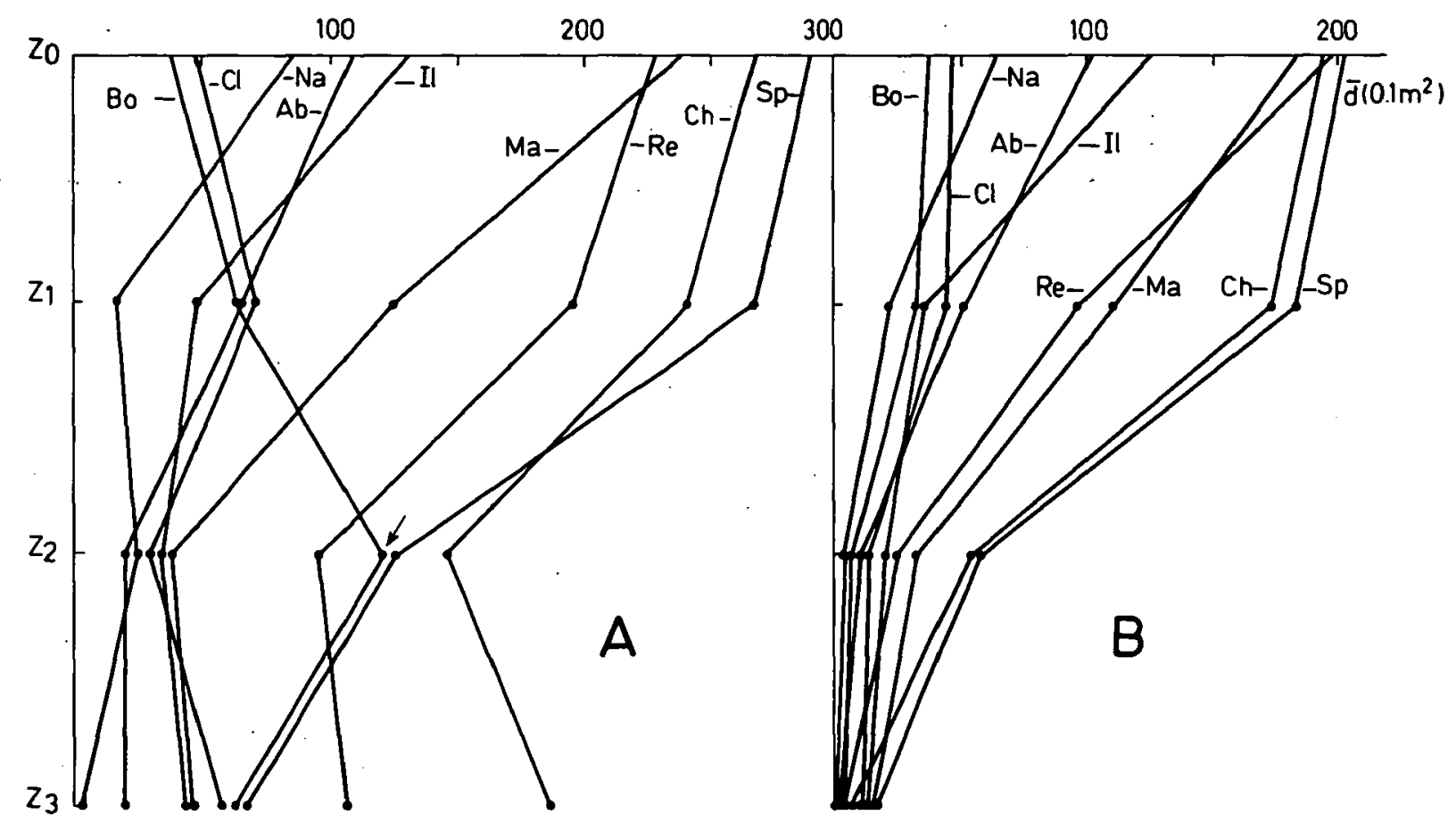

Fig. 8. Variation de l'abondance du macrobenthos en fonction de la profondeur relative - 9 lacs du Jura. A : faune globale - B : sauf Chaoborus, Tubificidae et Nématodes.

Fig. 8. Variation of the macrobenthos density in relation to the relative depth. 9 lakes of the Jura. A : total fauna. B : except Chaoborus, Tubificidae and Nematodes. 


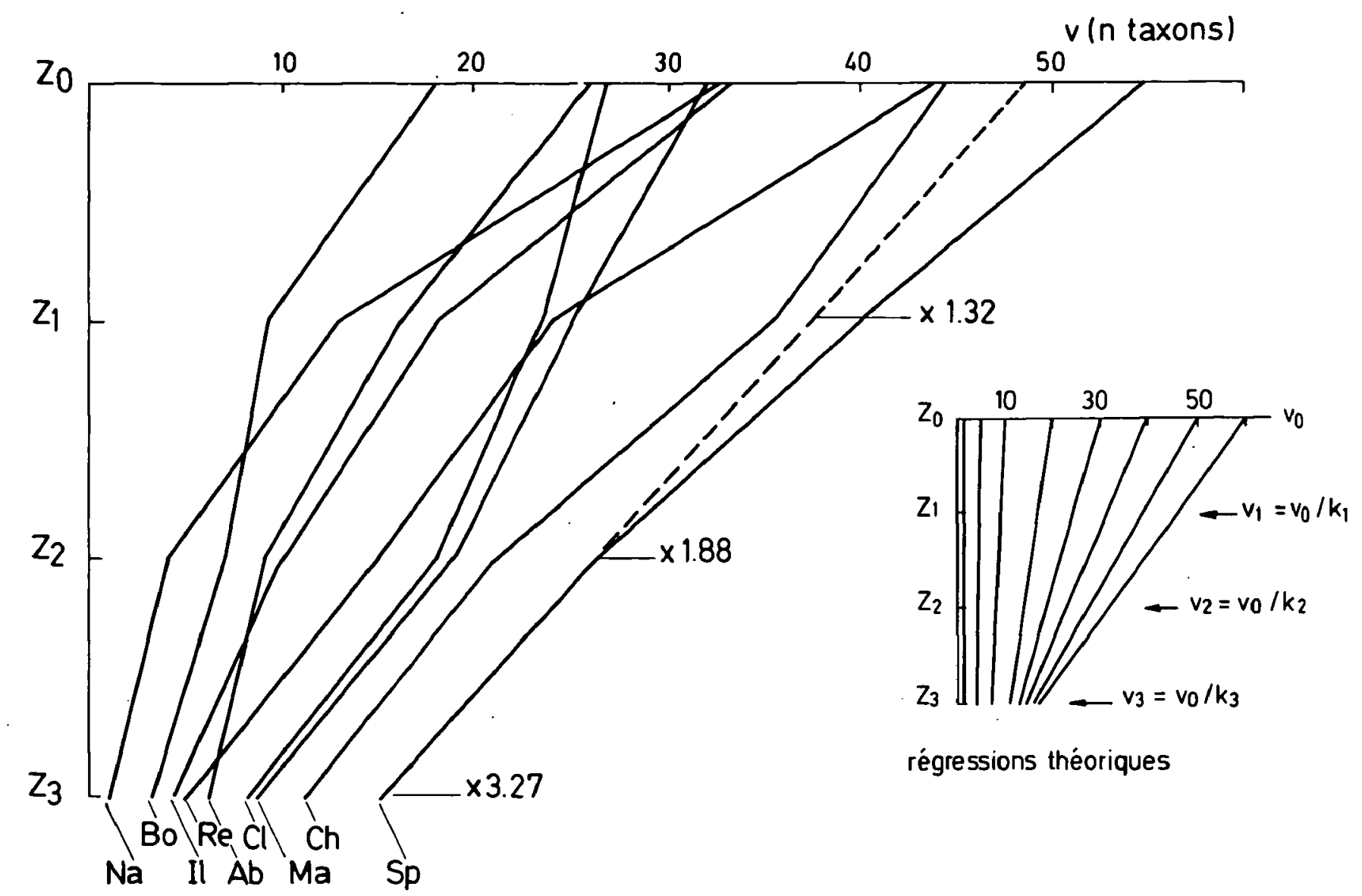

Fig. 9. Variation de la variété taxonomique en fonction de la profondeur relative de 9 lacs du Jura.

Fig. 9. Variation of the taxonomic variety in relation to the relative depth of 9 lakes of the Jura.

Parrish \& Wilhm (1978) ont montré que la diversité (indice de Shannon) pouvait également évoluer de façon linéaire selon la profondeur. Le fait que les lacs Maclu et Clairvaux présentent des régressions de ce type, bien que leurs variétés et densités soient nettement plus faibles, indique que les distributions de la variété en fonction de la profondeur traduit un mode de fonctionnement plutôt qu'un niveau trophique et suggère d'introduire un coefficient de correction du déficit taxonomique relatif.

\section{Correction du déficit taxonomique relatif}

Ce déficit relatif est, dans des conditions favorables, d'autant plus fort que la variété littorale (Vo) est grande (pentes plus faibles des régressions, Fig. 9).

Dans ces conditions, l'introduction d'un coefficient de correction $(k)$ permettra la comparaison des distributions verticales relatives en se référant à un système général dans lequel toutes les régressions seraient comprises entre une parallèle à l'axe des ordonnées, droite qui correspondrait à une variété littorale minimale de 1 genre, et la régression de la meilleure distribution observée. Cette dernière est représentée par celle du lac de St-Point dont la régression en zone profonde correspond au maintien de 15 taxons (soit $31 \%$ de la variété littorale) à $Z_{3}$ (soit $0,75 \mathrm{Zmax}$ ).

A cette régression théorique optimale, proche de celle observée du lac de St-Point, correspondent des multiplicateurs (m) de $1,32,1,88$ et 3,27 permettant l'obtention de $100 \%$ de la variété littorale $\left(V_{0}\right)$ aux profondeurs relatives successives $Z_{1}, Z_{2}$ et $Z_{3}$ (Fig. 9).

La relation $m=f(v o)$ permet de définir les coefficients de correction $k_{1}, k_{2}, k_{3}$ tels que $k=a x+$ 1 (avec $a=k_{z}-1 / V_{z}-1$ ) par lesquels il convient de multiplier toute valeur de $\mathrm{v}$ obtenue aux profondeurs relatives $Z_{1}, Z_{2}$ et $Z_{3}$. On obtient $k_{1}=0,006 v_{0}+$ $1, \mathrm{k}_{2}=0,018 \mathrm{v}_{\mathrm{o}}+1$ et $\mathrm{k}_{3}=0,047 \mathrm{v}_{\mathrm{o}}+1$.

Nota : il a été vérifié que, dans la gamme des relevés considérée, une relation linéaire s'établissait entre vo et les coefficients $k(r>0,80)$ mais il n'est pas exclu que l'analyse de systèmes plus oligotrophes, présentant des vo plus faibles, ne conduirait pas à une relation de type exponentiel.

Il a été vérifié également que les régressions linéaires prises comme références correspondaient effectivement à 
des biotopes sans déficit en oxygène (lacs de St. Point de $Z_{o}$ à $Z_{3}$, lacs de Châlain, Clairvaux et Maclu entre $Z_{o}$ et
$Z_{2}$. Les valeurs des coefficients $k$ obtenues par les formules précédentes figurent dans le tableau 8.

Tableau 8. Valeurs des coefficients de correction $\mathrm{k}$ et des indices de distribution faunistique $\mathrm{f}=\mathrm{kv} / \mathrm{vo}$ calculés pour les 9 lacs jurassiens étudiés.

Table 8. Values of the coefficient of correction $k$ and faunistic distribution index $f=k v / v o$ calculated for the 9 studied lakes of the Jura.

\begin{tabular}{|c|c|c|c|c|c|c|c|c|c|c|}
\hline Prof & & $A B$ & Bo & Ch & $\mathrm{Cl}$ & 11 & $\mathrm{Ma}$ & $\mathrm{Na}$ & $R_{e}$ & Sp \\
\hline 20 & vo & 26,00 & 18,00 & 44,00 & 27,00 & 33,00 & 32,00 & 33,00 & 44,00 & 54,00 \\
\hline $\mathbf{Z 1}$ & $k 1$ & 1,16 & 1,11 & 1,26 & 1,16 & 1,20 & 1,19 & 1,20 & 1,26 & 1,32 \\
\hline 22 & $k 2$ & 1,46 & 1.32 & 1,79 & 1,48 & 1,59 & 1,58 & 1,59 & 1,79 & 1,97 \\
\hline 23 & $\mathrm{~K} 3$ & 2,22 & 1,88 & 3,10 & 2,27 & 2,55 & 2,50 & 2,55 & 3,10 & 3,53 \\
\hline $\mathbf{Z 1}$ & $k 1-v 1$ & 19,00 & 10,00 & 45,00 & 28,00 & 22,00 & 30,00 & 16,00 & 30,00 & 52,80 \\
\hline $\mathbf{Z 2}$ & $k 2-v 2 \therefore$ & 13,00 & $\quad 9,00$ & 38,00 & 27,00 & 16,00 & 30,00 & 6,00 & 27,00 & 53,19 \\
\hline 23 & $k 3-v 3$ & 13,00 & 6,00 & 34,00 & 18,00 & 10,00 & 20,00 & 3,00 & 16,00 & 52,95 \\
\hline 21 & $\$ 1$ & 0,73 & 0,55 & 1,00 & 1,00 & 0,67 & 0,94 & 0,48 & 0,68 & 0,97 \\
\hline 22 & $f 2$ & 0,50 & 0,50 & 0,86 & 1,00 & 0,48 & 0,93 & 0,18 & 0,61 & 0.98 \\
\hline \multirow[t]{2}{*}{23} & 13 & 0,50 & 0,33 & 0,77 & 0,67 & 0.30 & 0.62 & 0,09 & 0,36 & 0,98 \\
\hline & $t$ & 0,58 & 0,46 & 0,88 & 0,88 & 0,48 & 0,83 & 0,25 & 0,55 & 0,98 \\
\hline
\end{tabular}

Ces coefficients permettent de définir les indices de distribution faunistique $f_{1}, f_{2}$ et $f_{3}$, tels que $f=$ k.v/vo. Les variations de l'indice $f$ en fonction de la profondeur relative sont portées sur la figure 10 .

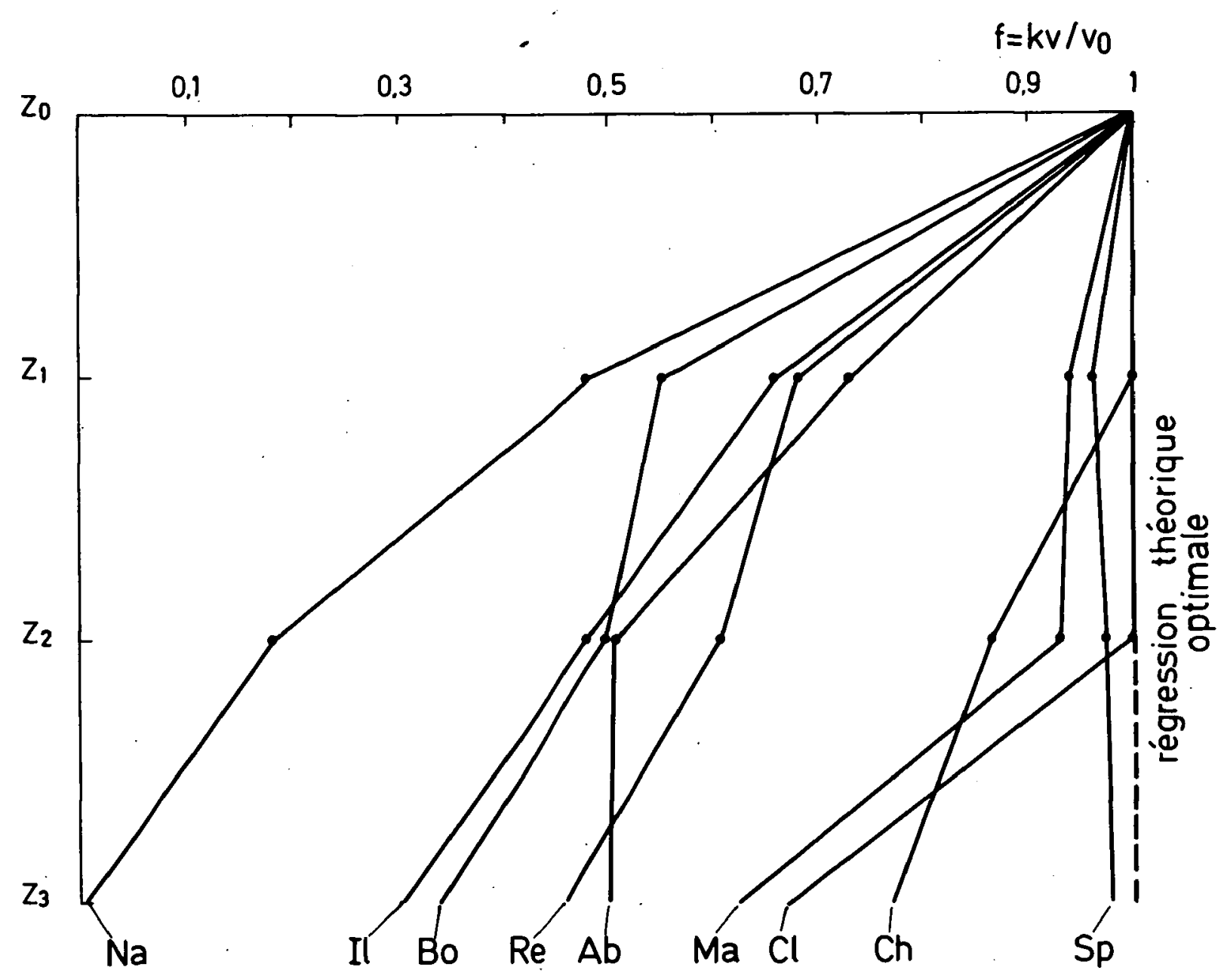

Figure 10. Distributions faunistiques verticales relatives comparées d'après les indices de distribution faunistique - Macrobenthos de 9 lacs du Jura.

Fig. 10. Comparative vertical faunistic relative distributions from the faunistic distribution indexes. Macrobenthos of 9 lakes of the Jura. 
Les distributions des indices $f$ s'interprètent par rapport à une distribution optimale représentée par la verticale de $f=1$. On note que les distributions les plus proches de cet optimum théorique concernent les lacs de St. Point de $Z_{0}$ à $Z_{3}$ de Clairvaux et Maclu jusqu'à $Z_{2}$ et de Châlain jusqu'à $Z_{1}$.

\subsection{Seconde classification des lacs}

Un classement des lacs étudiés peut alors être effectué en fonction des deux composantes précédemment définies : un indice biologique littoral : $B_{0}$ $v_{0} . \sqrt{d_{o}}\left(\operatorname{avec} d_{o}=n\right.$ individus $\left./ 0,1 \mathrm{~m}^{2}\right)$ et les indices de distribution bathymétrique de la faune (f) obtenus aux profondeurs $Z_{1}, Z_{2}$ et $Z_{3}$.

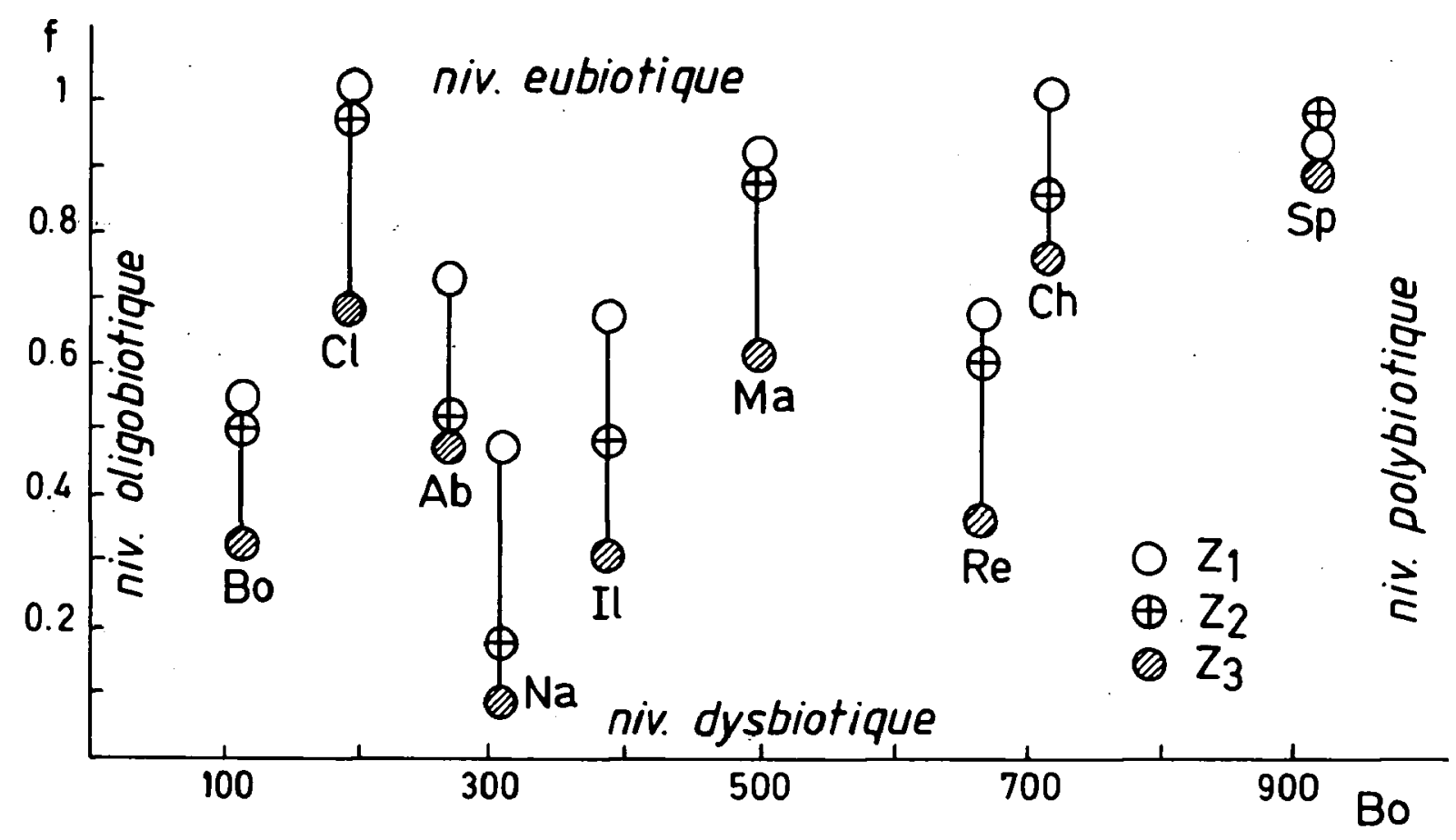

Fig. 11. Classification de 9 lacs du Jura d'après l'indice biologique littoral (Bo) et l'indice de distribution faunistique (f) - macrobenthos des sédiments - 650 prélèvements.

Fig. 11. Classification of the 9 lakes of the Jura using the littoral biological index (Bo) and the faunistic distribution index (f). Macrobenthos of the sediments. 650 samples.

On retrouve les grandes lignes du classement des systèmes obtenus précédemment (Fig. 7), mais cette fois les lacs peu biogènes (Clairvaux, Narlay, Ilay) sont différenciés par les indices de distribution faunistique (f) et cette classification est très proche de celle fournie par l'A.F.C. globale (Fig. 5).

\section{- Interprétation-classement des lacs}

Les lacs se distribuent en fonction d'un critère quantitatif $\left(B_{0}\right)$, exprimant l'aptitude du système à la production secondaire dans des conditions optimales (zone littorale, euphotique, non désoxygénée) et d'un critère qualitatif (f) exprimant de façon synthétique la structure physico-chimique verticale du lac.

Dans le système de classement proposé deux niveaux expriment des résultantes d'ordre qualitatif : les lacs de bonne qualité biologique ou lacs eubiotiques (de $e u=$ bien, vrai) sont opposés aux lacs de mauvaise qualité biologique ou lacs dysbiotiques (de $d u s=$ en difficulté, perturbé).

Les indications des deux autres niveaux (Fig. 11) sont d'ordre quantitatif : les lacs oligobiotiques (avec peu de faune) sont opposés aux lacs polybiotiques (avec beaucoup de faune) comme un lac oligohumique s'oppose à un lac polyhumique (en effet poly du grec polus signifie à la fois nombreux et beaucoup). Le lac de St-Point, présentant la valeur biogénique optimale, est à la fois polybiotique et eubiotique et à l'opposé, le lac de Bonlieu est à la fois oligobiotique et dysbiotique.

\section{Conclusion}

L'analyse comparative des peuplements des sédiments de 9 lacs du Jura, réalisée selon un protocole d'échantillonnage précis fondé sur des éléments 
relatifs (profondeurs relatives, nombres d'échantillons relatifs à la longueur des isobathes) permet, sans référence à la nature des taxons, de proposer un classement des lacs dans un système d'axes où figurent en ordonnée un indice de distribution faunistique et en abscisse un indice biologique littoral.

Les résultats obtenus se révèlent cohérents par rapport aux résultats obtenus antérieurement sur les mêmes systèmes (CEMAGREF 1982-1987) et des correspondances générales s'établissent entre ce classement et celui obtenu à partir des descripteurs physiques et chimiques de l'eau et des sédiments (Verneaux et al. 1987-1991, monographies lacustres, op. cit.).

Les lacs de St-Point, Châlain, Clairvaux, proches du niveau eubiotique contiennent peu de matière organique (MO $<10 \%$ ) dans leurs sédiments essentiellement carbonatés (craie lacustre). A l'opposé, les lacs de Bonlieu, l'Abbaye et Ilay contiennent beaucoup plus de matière organique résiduelle (MO $>25 \% \mathrm{~ms}$ ) et la désoxygénation de l'hypolimnion y est accentuée. Le lac de Narlay, très proche du niveau dysbiotique à $3 / 4 \mathrm{Zmax}$, est un lac pollué par des eaux résiduelles karstiques profondes.

Il serait toutefois prématuré de proposer une interprétation écologique de la classification résultante obtenue. Ce passage de la phase descriptive à la phase interprétative repose sur un examen préalable des correspondances entre les deux séries d'analyses, biologiques et mésologiques.

L'hypothèse de travail retenue est que l'indice littoral exprimerait surtout un potentiel biogénique alors que l'indice de distribution faunistique correspondrait davantage au mode de fonctionnement du système considéré.

On observe d'autre part qu'un lac très pollué, qui de ce fait ne possèderait que des taxons euryèces en zone littorale, est théoriquement susceptible d'occuper dans le graphe (Fig. 11) la même position qu'un lac oligotrophe, à la fois oligobiotique et eubiotique. Le critère nature de la faune est alors à prendre en considération pour effectuer la discrimination de ces deux types extrêmes de lacs oligobiotiques.

Les recherches se poursuivent, dans deux directions, visant à relier les organisations biologiques aux complexes écologiques : l'analyse des relations entre le classement proposé et des descripteurs des milieux concernés (eau et sédiments) ainsi que celle des correspondances susceptibles d'être établies entre la classification obtenue, la nature des taxons et les typologies proposées par d'autres auteurs.

\section{Remerciements}

Les auteurs remercient J.P. Massonie, pour sa contribution au traitement mathématique des données et $\mathrm{H}$. Gimaret et J.C. Lambert pour leur collaboration aux missions sur le terrain et à l'analyse des échantillons.

\section{Travaux cités}

Bagge P. 1969. - Ecological studies on the fauna of subarctic waters in Finnish Lapland. Annls Univ. turku A, II : 28-76.

Benzecri J.P. 1973. - L'analyse des données. I : la Taxonomie, 615 p. II : L'analyse des correspondances, Dunod éd., Paris : $620 \mathrm{p}$.

Bonomi G. \& Ruggiu D. 1966. - II Macrobenton profondo del Lago di Mergozzo. Mémorie Ist. Ital. Idrobiol., 20 : 153-200.

Brinkhurst O.R. 1969. - Changes in the benthos of lakes Erie and Ontario. Bull. Buffalo Soc. nat. Sci., 25 : 45-65.

Brinkhurst O.R. 1974. - The Benthos of Lakes. Mc Millan Press. Ltd, London and Basingstoke : $190 \mathrm{p}$.

Brundin L. 1949. - Chironomiden and andere Bodentiere der Südschwedischen Urgebirgsseen. Rep. Inst. Freshwat. Res. Drottningholm, 30 : 1-914.

Brundin L. 1958. - The bottom faunistical lake type system and its application to the southern hemisphere. Moreover a theory of glacial erosion as a factor of productivity in lakes and oceans. Verh. int. Ver. Limnol., 13 : 288-297.

CEMAGREF in SRAE France-Comté. 1982-1987. Monographies des lacs du Jura L'Abbaye, 1982 : 61 p. et annexes - Clairvaux, 1984 : 99 p. et annexes - Maclu, 1985 : 104 p. et annexes - Châlain, $1986: 116$ p. et annexes - Ilay, 1986 : 95 p. et annexes - Bonlieu, $1987: 97$ p. et annexes Etival, ${ }_{0} 1987: 87$ p. et annexes - SRAE Fr. Comté, éd. Besançon.

Cummins K.W. 1962. - An evaluation of some techniques for the collection and analysis of benthic samples with special emphasis on lotic waters. Am. Midl. Nat., 67 : 447-504.

Downing J.A. \& Rigler F.H. 1984. - A Manual of Methods for the Assessment of Secondary Productivity in Fresh Waters. IBP. Hand Book, 17, Blackwell Scient. Publ., Oxford : $476 \mathrm{p}$

Elliott J.M. 1977. - Some methods for the Statistical Analysis of samples of Benthic Invertebrates 2nd ed. Scient. Publs Freshwat. biol. Ass., 25; $156 \mathrm{p}$.

Gaufin A.R., Harris E.K. \& Walter H.J. 1956. - A statistical evaluation of stream bottom sampling data obtained from three standard samplers. Ecology, 37 (4) : 643-648.

Giani N. \& Lucas C. 1974. - Les sédiments d'un lac de haute montagne : structure, nature et peuplement. Annis Limnol., 10 (53) : 223-244.

Hakanson L. 1981. - Sjösedimenten $i$ recipientkontrollen; principer, processer och praktiska exempel. Natl. Swed. Environ. Prot. Board, SNV PM 1398, Uppsala : 242 p.

Humphries C.F. 1936. - An investigation of the profundal and sub-littoral fauna of Windermere. J. anim. Ecol., $5: 29-52$. 
Jónasson P.M. 1961. - Population dynamics in Chironomus anthracinus Zett. in the profundal zone of Lake Esrom. Verh. int. Ver. Limnol., $14:$ 196-203.

Juget J. 1958. - Recherches sur la faurie de fond du Léman et du lac d'Annecy. Ann. Stat. C. Hydrobiol. appl., 7 : 9-94.

Laboratoire d'Hydrobiologie, Université de Besançon 1979. Etude écologique des lacs de St. Point et de Remoray. Univ. Fr. Comté, Lab. Hydrobiol. éd., 105 p. + annexes.

Lafont M. 1989. - Contribution à la gestion des eaux continentales : utilisation des Oligochètes comme descripteurs de l'état biologique et du degré de pollution des eaux et des sédiments. Thèse Doc. Etat, Univ. Cl. Bernard Lyon I : 287 p.

Laville H. 1971. - Recherches sur les Chironomides (Diptera) lacustres du Massif de Néouvielle (Hautes-Pyrénées). Annls Limnol., 7 (2) : 173-332.

Lündbeck J. 1926. - Die Bodentierwelt Norddeutscher Seen. Arch. Hydrobiol. Suppl., 7 : 1-473.

Lündbeck J. 1936. - Untersuchungen über die Bodenbesiedlung der Alpenrandseen Arch. Hydrobiol. Suppl., 10 : 208-358.

Macan T.T. 1970. - Biological Studies of the English Lakes. Longman Group Ltd ed. London : 260 p.

Milbrink G. 1978. - Indicator communities of Oligochaetes in Scandinavian lakes. Verh. int. Ver. Limnol., 20 : 2406-2411.

Mouthon J. 1986. - Principes généraux pour une méthode d'appréciation de la qualité globale des sédiments lacustres à l'aide d'une analyse simplifiée des malacocénoses. Annls Limnol., 22 (3) : 209-217.

Oliver D.R. 1960. - The Macroscopic Bottom. Fauna of Lac la Ronge, Saskatchewan. J. Fish. Res. Bd Canada., 17 (5) : 607-624.

Parrish J.H. \& Wilhm J. 1978. - Relationship between physicochemical conditions and the distribution of benthic macroinvertebrates in Arbuckle Lake. The S. West. Nat., 23 (1) : 135-144.

Prat N. 1978. - Benthos typology of Spanish reservoirs. Verh. int. Ver. Limnol., 20 : 647-1651.
Rawson D.S. 1953. - The Bottom Fauna of Great Slave Lake. J. Fish Res. Bd Canada., 10 (8) : 486-520.

Ricou G. 1967. - Etude biocénotique d'un milieu « naturel » : la prairie permanente pâturée. Annls Epiphytics, 18 (1) : 1-148.

Rofes G. \& Savary R. 1981. - Description d'un nouveau modèle de carottier pour sédiments fins. Bull. fr. Piscic., 283 : 102-113.

Saether O.A. 1975. - Nearctic chironomids as indicators of lake typology. Verh. int. Ver. Limnol., 19 : 3127-3133.

Slack H.D. 1965. - The profundal Fauna of Loch Lomond, Scotland. Proc. R. Soc., Edinb., 65 (6) : 272-297.

Schwoerbel J. 1966. - Methoden der Hydrobiologie. G. Fisher Verlag, Stuttgart : $207 \mathrm{p}$.

Thienemann A. 1913. - Der Zusammenhang zwischen dem Sauerstoffgehalt des Tiefenwassers und der Zusammensetzung der Tierfauna unserer Seen. Int. Revue ges. Hydrobiol., $6: 243-249$

Thienemann A. 1931. - Tropische Seen und Seetypenlehrie. Arch. Hydrobiol. Suppl. 9 : 205-231.

Verneaux J., Remy F., Vidonne A. \& Guyard A. 1987. Caractères généraux des sédiments de 10 lacs jurassiens. Rev. Sci. de l'eau, 6 : 107-128.

Verneaux J., Guyard A., Joly D. \& Vidonne A. 1988. - Etude physico-chimique des sédiments du lac de St. Point (massif du Jura) : cartographie thématique. Annls Limnol., 24 (1) : 15-29.

Verneaux J., Vidonne A., Remy F., Guyard A. 1991. Particules organiques et rapport $\mathrm{C} / \mathrm{N}$ des sédiments des lacs du Jura. Annls Limnol., 4 (2) : 175-190.

Wasson J.G. 1984. - L'utilisation des peuplements larvaires de Chironomidae (Diptera) pour la diagnose écologique des lacs : essai d'application aux deux lacs de Clairvaux (Jura, France), Rev. Sci. de l'eau, $3:$ 395-408.

Wiederholm T. \& Eriksson L. 1977. - Benthos of an acid lake. Oikos, 29 : 261-267. 